\title{
Six Lonely Runners
}

\author{
Tom Bohman * \\ Department of Mathematics \\ Massachusetts Institute of Technology \\ tbohman@moser.math.cmu.edu
}

\author{
Ron Holzman ${ }^{\dagger \ddagger}$ \\ Department of Mathematics \\ Technion - Israel Institute of Technology \\ holzman@tx.technion.ac.il
}

\section{Dan Kleitman}

Department of Mathematics

Massachusetts Institute of Technology

djk@math.mit.edu

Submitted: March 8, 2000; Accepted: February 6, 2001.

MR Subject Classifications: 11B75, 11J71

\begin{abstract}
For $x$ real, let $\{x\}$ be the fractional part of $x$ (i.e. $\{x\}=x-\lfloor x\rfloor$ ). In this paper we prove the $k=5$ case of the following conjecture (the lonely runner conjecture): for any $k$ positive reals $v_{1}, \ldots, v_{k}$ there exists a real number $t$ such that $1 /(k+1) \leq$ $\left\{v_{i} t\right\} \leq k /(k+1)$ for $i=1, \ldots, k$.
\end{abstract}

\section{Introduction}

Consider the following problem. There are $n$ people running on a circular track of circumference 1 . All $n$ runners start at the same time and place. It is not a race; runner $i$ runs at constant speed $v_{i}$. Thus, the position of runner $i$ at time $t$ is $\left\{v_{i} t\right\}$ where $\{x\}$ is the fractional part of $x$ (i.e. $\{x\}=x-\lfloor x\rfloor$ ). All the speeds are different (i.e. $v_{i} \neq v_{j}$ for $i \neq j$ ). A runner is said to be lonely if the smallest distance (along the track) to another runner is at least $1 / n$. To be precise, runner $i$ is lonely at time $t$ if the following holds:

$$
\left\{v_{i} t-v_{j} t\right\} \in[1 / n,(n-1) / n] \text { for all } j \neq i .
$$

\footnotetext{
*Research supported by NSF Grant DMS-9627408

${ }^{\dagger}$ Research supported by the M. and M. L. Bank Mathematics Research Fund and by the Fund for the Promotion of Research at the Technion.

${ }^{\ddagger}$ Work partly done while this author was visiting the Department of Mathematics, Massachusetts Institute of Technology.
} 
For example, if there are exactly two runners on the track then there comes a time when they are opposite each other, and at this moment both runners are lonely. The question: does every runner get lonely?

This question originally arose in the context of diophantine approximations (see [BW], $[\mathrm{W}]$ ) and in the study of so-called View Obstruction Problems (see [C1], [C2], [C3]). It has been shown that if there are less than or equal to five runners on the track then every runner gets lonely [BGGST], [CP]. In [BGGST], this result was used to prove a theorem on flows in graphs related to Seymour's six-flow theorem [S]. Furthermore, it was pointed out that a proof of the lonely runner conjecture for higher values of $n$ would have analogous consequences regarding flows in regular matroids.

While the formulation of the question in the above paragraph (due to Goddyn [BGGST]) is poetic, the following reformulation of the problem will be easier to handle.

Conjecture 1 (Wills, Cusick). For any collection $v_{1}, v_{2}, \ldots, v_{n-1} \in \mathbb{R}^{+}$there exists $t \in \mathbb{R}^{+}$such that the following holds:

$$
\left\{v_{i} t\right\} \in[1 / n,(n-1) / n] \text { for } i=1, \ldots, n-1 .
$$

To get this conjecture from the original simply choose an $i$ and subtract $v_{i}$ from each of the original speeds. After doing so, runner $i$ is standing still and is lonely at time $t$ if all the other runners are far from the starting point at time $t$ (i.e. far from 0 ). Condition (1) holds for time $t$ if and only if the original runner $i$ is lonely at time $t$.

The following example shows that Conjecture 1 is sharp. Let $v_{i}=i$ for $i=1, \ldots, n-1$, and assume for the sake of contradiction that there exists a time $t$ for which $\left\{v_{i} t\right\} \in$ $(1 / n,(n-1) / n)$ for all $i$. Then there exist $i$ and $j$ such that $\left\{v_{i} t\right\} \leq\left\{v_{j} t\right\}<\left\{v_{i} t\right\}+1 / n$. However, $v_{j}-v_{i}=v_{k}$ for some $k \in\{1, \ldots, n-1\}$ (note that for the purposes of this problem $v_{i}$ and $-v_{i}$ are equivalent speeds) and $\left\{v_{k} t\right\}=\left\{v_{j} t\right\}-\left\{v_{i} t\right\}<1 / n$. This is a contradiction. It should be noted that a number of other, sporadic extremal examples have been discovered for particular values of $n$.

Before stating our central result, we give a third formulation of the problem, a restatement of the conjecture as a covering problem. Define

$$
B=\left\{x \in \mathbb{R}^{+}: \exists k \in \mathbb{Z} \text { such that }|k-x|<1 / n\right\}
$$

and $x_{i}=1 / v_{i}$ for $i=1, \ldots, n-1$. Runner $i$ is near the imaginary stationary runner (i.e. near the starting point) for $t \in B_{i}:=x_{i} B$. Thus, there exists $t$ satisfying condition (1) if and only if we have

$$
\mathcal{B}:=\bigcup_{i=1}^{n-1} x_{i} B \neq \mathbb{R}^{+}
$$

Thus, condition (1) is equivalent to the statement that no set of $n-1$ contractions and/or expansions of $B$ covers $\mathbb{R}^{+}$. Throughout the remainder of the paper we will pass between the formulation of the problem given by (1) and that given by (2) without comment.

Conjecture 1 has been proven for $n \leq 5[\mathrm{CP}]$, [BGGST]. Here we prove that the conjecture holds for $n=6$. 
Theorem 2. For any collection $v_{1}, v_{2}, v_{3}, v_{4}, v_{5} \in \mathbb{R}^{+}$there exists $t \in \mathbb{R}^{+}$satisfying

$$
\left\{v_{i} t\right\} \in[1 / 6,5 / 6] \text { for } i=1, \ldots, 5 \text {. }
$$

Now, the arguments used in [CP] and [BGGST] for the proof of Conjecture 1 for $n \leq 5$ rely on number theoretical analyses of the speeds (which are assumed to be integers) focusing on how the runners cover discrete sets of times. In contrast, the proof we give here takes advantage of the fact that runners must cover intervals of times. Because of this difference in approach, we are also able to show that there are, up to scaling, only two sets of speeds for which $\overline{\mathcal{B}}=\mathbb{R}^{+}$; in other words, there are two extremal examples. The second of these is one of the sporadic extremal examples found by Flor (see $[W]$ ).

Theorem 3. Let $v_{1}<v_{2}<v_{3}<v_{4}<v_{5}$ be a collection of positive reals. Either $\left(v_{1}, \ldots, v_{5}\right)=x(1,2,3,4,5)$ for some $x \in \mathbb{R}^{+},\left(v_{1}, \ldots, v_{5}\right)=y(1,3,4,5,9)$ for some $y \in$ $\mathbb{R}^{+}$or there exists a time $t$ and an $\epsilon>0$ satisfying

$$
[t, t+\epsilon] \cap \mathcal{B}=\emptyset
$$

The methods developed in this paper can certainly be used to prove statements analogous to Theorems 2 and 3 for $n<6$. For the sake of brevity, a discussion of such arguments is not included here. The same methods may be used to attack the problem for $n>6$, but the amount of work involved seems to grow so fast with $n$ as to make this approach impractical.

\section{The Argument}

We begin with some preparatory assumptions and definitions. We first assume $v_{1}, \ldots, v_{5}$ are rational. This assumption is justified by Lemma 8, which is stated and proved in Section 4. For notational convenience we assume $v_{1}<v_{2}<v_{3}<v_{4}<v_{5}$ and $v_{3}=1$ (in other words $x_{1}>x_{2}>x_{3}>x_{4}>x_{5}$ and $x_{3}=1$ ). For $S \subseteq\{1,2,3,4,5\}$, a maximal interval contained in $\cup_{i \in S} B_{i}$ is called a $S$-block and a maximal interval contained in $\mathbb{R}^{+} \backslash\left(\cup_{i \in S} B_{i}\right)$ is called a $S$-gap. Note that $S$-gaps are closed intervals and $S$-blocks are open intervals. For notational convenience we will drop brackets and commas when discussing $S$-blocks and $S$-gaps; for example, we will write 45 -gap instead of $\{4,5\}$-gap. The length of an interval $I$ will be denoted $|I|$.

The starting point for our argument is the following simple observation.

Lemma 4. If $I$ is a 45-block then $|I|<2 x_{4} / 3$.

Proof. First note that $I$ contains at most one 4-block because a 4-gap (which has length $2 x_{4} / 3$ ) cannot be contained in a 5 -block (which has length $x_{5} / 3<x_{4} / 3$ ). Let $I$ be the union of at most one 4 -block and $k 5$-blocks.

If $k \leq 1$ then $|I|<x_{4} / 3+x_{5} / 3<2 x_{4} / 3$.

Suppose $k \geq 2$. In this case $I$ contains a 5 -gap. This 5 -gap is a subset of some 4 -block $J$. Therefore, $2 x_{5} / 3<x_{4} / 3$. There are at most two 5 -blocks that are contained in $I$ but not contained in $J$. Thus, $|I|<x_{4} / 3+2 x_{5} / 3<2 x_{4} / 3$. 
Corollary 5. If there exists a 3-gap that is also a 123-gap then $\mathcal{B} \neq \mathbb{R}^{+}$.

Proof. Let $I$ be a 3 -gap that is also a 123 -gap. Since $I$ is a 3 -gap, $|I|=2 x_{3} / 3>2 x_{4} / 3$. Thus, $I$ is contained in no 45 -block, and $I \nsubseteq \mathcal{B}$.

In this section we establish sufficient conditions for the existence a 3-gap that is also a 123-gap. Later in the paper we handle collections of speeds that fail to satisfy these sufficient conditions case by case. The machinery developed in this section will be used repeatedly when we consider the special cases.

When does there exist a 3-gap that is also a 123-gap? Consider an arbitrary 3-gap $I$. It is an interval of time when runner 3 is in the interval $[1 / 6,5 / 6]$. The interval $I$ is also a 23-gap if and only if runner 2 is also in $[1 / 6,5 / 6]$ throughout $I$, which is the case if and only if runner 3 passes runner 2 somewhere in $I$. Now, the times when runner 3 passes runner 2 are the positive integer multiples of $t_{0}$, where $t_{0}$ is defined by

$$
t_{0}:=\frac{1}{v_{3}-v_{2}}=\frac{1}{1-v_{2}} .
$$

If we have

$$
\left\{v_{3} k t_{0}\right\}=\left\{k t_{0}\right\} \in[1 / 6,5 / 6]
$$

then runner 3 is in $[1 / 6,5 / 6]$ at time $k t_{0}$, and $k t_{0}$ is in a 3 -gap that is also a 23-gap. This 23-gap is also a 123-gap if and only if the following three conditions are satisfied:

1. runner 1 is in $[1 / 6,5 / 6]$ at time $k t_{0}$ :

$$
\left\{v_{1} k t_{0}\right\} \in[1 / 6,5 / 6],
$$

2. the last time before $k t_{0}$ that runner 3 enters $[1 / 6,5 / 6]$ follows the last time before $k t_{0}$ that runner 1 enters $[1 / 6,5 / 6]$ :

$$
\frac{\left\{v_{1} k t_{0}\right\}-1 / 6}{v_{1}} \geq \frac{\left\{k t_{0}\right\}-1 / 6}{1}
$$

and

3. the first time after $k t_{0}$ that runner 3 leaves $[1 / 6,5 / 6]$ precedes the first time after $k t_{0}$ that runner 1 leaves $[1 / 6,5 / 6]$ :

$$
\frac{5 / 6-\left\{v_{1} k t_{0}\right\}}{v_{1}} \geq \frac{5 / 6-\left\{k t_{0}\right\}}{1} .
$$

Conditions 2 and 3 are equivalent to

$$
1 / 6+\left(\left\{k t_{0}\right\}-1 / 6\right) v_{1} \leq\left\{v_{1} k t_{0}\right\} \leq 5 / 6-\left(5 / 6-\left\{k t_{0}\right\}\right) v_{1} .
$$

Thus, there exists a 3-gap that is also a 123-gap if and only if there exists a positive integer $k$ satisfying (4), (5) and (6). 
In order to get a better feel for this, we restate these conditions in slightly different language. Let $G \subseteq T:=[0,1) \times[0,1)$ be defined by

$$
G=\left\{\left(\left\{k t_{0}\right\},\left\{v_{1} k t_{0}\right\}\right): k=0,1, \ldots\right\} .
$$

Note that the rationality of $v_{1}$ and $v_{2}$ implies that $G$ is finite. Also note that $G$ is merely the subgroup of $T$, with respect to addition modulo 1 in each coordinate, generated by $\left(\left\{t_{0}\right\},\left\{v_{1} t_{0}\right\}\right)$. Now, let $P$ be the collection of $(x, y) \in T$ satisfying

$$
\begin{gathered}
1 / 6 \leq x \leq 5 / 6, \text { and } \\
\left(1-v_{1}\right) / 6+v_{1} x \leq y \leq 5\left(1-v_{1}\right) / 6+v_{1} x .
\end{gathered}
$$

There exists a 3-gap that is also a 123-gap if and only if $G \cap P \neq \emptyset$ (note that (5) follows from (8) and (9)).

This formulation of our problem leads naturally to the question: under what conditions does a finite cyclic subgroup of the two dimensional torus intersect a given polygon lying on the torus? It seems reasonable to think that if the polygon is sufficiently large then such an intersection will exist when $G$ is 'random looking;' that is, the intersection is nonempty so long as $G$ doesn't follow some very restrictive pattern (e.g. $G$ lies on a coordinate axis). This is in fact the case when the polygon in question is a rectangle with sides parallel to the coordinate axes, as is seen in the lemma below. Before stating this technical lemma we must establish some definitions. As above, let $T=[0,1) \times[0,1)$ be the two-dimensional torus. We shall sometimes specify a point $(x, y) \in T$ using values of $x, y$ which are not in $[0,1)$ - these should be understood modulo 1 . Let $G$ be an arbitrary finite subgroup of $T$. Define

$$
\begin{gathered}
N_{1}=\{x: \exists y \text { such that }(x, y) \in G\}, \\
N_{2}=\{y: \exists x \text { such that }(x, y) \in G\}, \\
n_{1}=\left|N_{1}\right|, \quad n_{2}=\left|N_{2}\right| \quad \text { and } n=|G| .
\end{gathered}
$$

Note that $N_{1}=\left\{i / n_{1}: i=0, \ldots, n_{1}-1\right\}, N_{2}=\left\{i / n_{2}: i=0, \ldots, n_{2}-1\right\}, n$ is a common multiple of $n_{1}, n_{2}$ (if $G$ is cyclic, it actually equals $\operatorname{lcm}\left\{n_{1}, n_{2}\right\}$ ), and $G \subseteq\{(i / n, j / n)$ : $0 \leq i, j \leq n-1\}$. A rectangle in $T$ is a set of the form

$$
R:=\left\{(u, v) \in T: 0 \leq\left\{u-x_{1}\right\} \leq \alpha \text { and } 0 \leq\left\{v-x_{2}\right\} \leq \beta\right\}
$$

for some $x=\left(x_{1}, x_{2}\right) \in T$, width $\alpha$, and height $\beta$. 


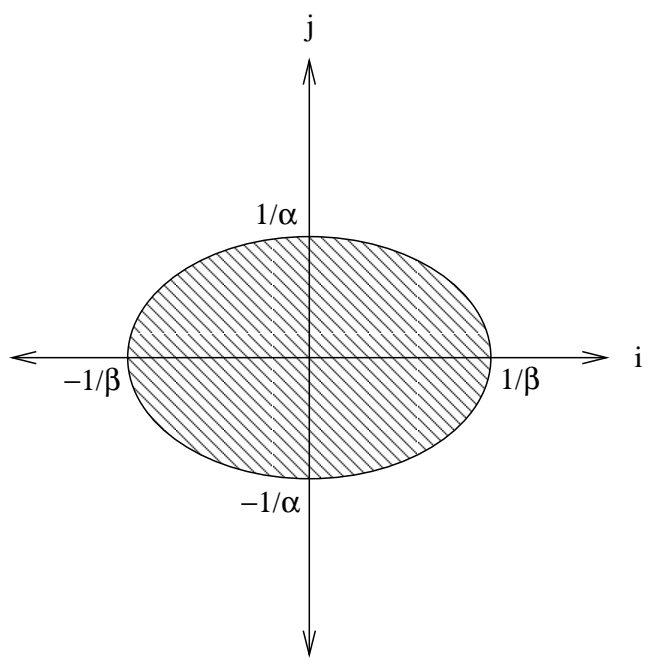

(a) $\quad \alpha \beta \geq \frac{2}{n}$

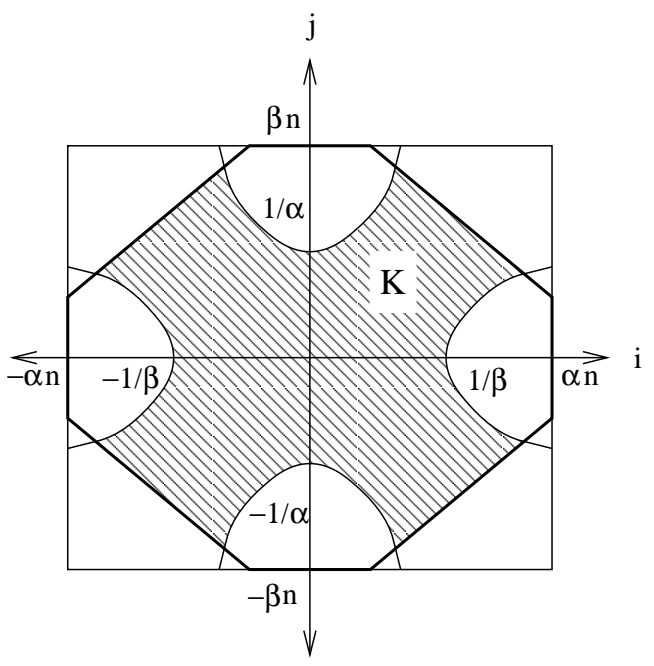

(b) $\frac{1}{n} \leq \alpha \beta<\frac{2}{n}$

Figure 1: The conditions in the main lemma. The region in $\mathbb{Z}^{2}$ which must contain an element $(i, j) \neq(0,0)$ such that $(i / n, j / n) \in G$ is shaded. The polygon with bold boundary in (b) is an example of a possible choice of $K$.

Main Lemma. Let $G$ be a finite subgroup of the torus $T$ of order $|G|=n$. Let $0<$ $\alpha, \beta \leq 1$ be given, and suppose $\alpha \beta \geq 1 / n$. Then $G$ intersects every rectangle $R$ in $T$ of width $\alpha$ and height $\beta$, unless one of the following conditions holds:

1. $\alpha \beta \geq 2 / n$ and there exists $(i, j) \in \mathbb{Z}^{2} \backslash\{(0,0)\}$ in the box $(-1 / \beta, 1 / \beta) \times(-1 / \alpha, 1 / \alpha)$ satisfying

$$
(i / n, j / n) \in G \quad \text { and } \quad \beta|i|+\alpha|j|-\frac{2}{n}|i||j|<1 .
$$

2. $1 / n \leq \alpha \beta<2 / n$ and for every symmetric closed convex neighborhood $K$ of the origin in $\mathbb{R}^{2}$ which is contained in the box $[-\alpha n, \alpha n] \times[-\beta n, \beta n]$ and has area $A(K) \geq 4 n$, there exists $(i, j) \in K \cap \mathbb{Z}^{2} \backslash\{(0,0)\}$ satisfying

$$
(i / n, j / n) \in G \quad \text { and } \quad \beta|i|+\alpha|j|-\frac{2}{n}|i||j|<1 .
$$

Note that when the area $\alpha \beta$ of the rectangles in question is less than $1 / n$, it is impossible for the group $G$ of order $n$ to intersect all of them. Hence, we restrict attention to $\alpha \beta \geq 1 / n$. We chose to distinguish the cases $\alpha \beta \geq 2 / n$ and $1 / n \leq \alpha \beta<2 / n$ in the statement of the lemma because when $n$ is large enough so that $\alpha \beta \geq 2 / n$ the condition in the lemma admits a simpler form. Figure 1 illustrates the condition in each of the two cases.

When applying the Main Lemma we will use the following simpler form.

Corollary 6. Let $G$ be a finite subgroup of the torus $T$ of order $|G|=n>1$. Let $0<\alpha, \beta<1$ be fixed. Either $G$ intersects every rectangle $R$ in $T$ of width $\alpha$ and height $\beta$ 
or there exists $(i, j) \in \mathbb{Z}^{2} \backslash\{(0,0)\}$ satisfying

$$
0 \leq i<\frac{1}{\beta}, \quad-\frac{1}{\alpha}-\frac{\beta}{\alpha} i<j<\frac{1}{\alpha}+\frac{\beta}{\alpha} i, \quad \text { and } \quad(i / n, j / n) \in G .
$$

The proofs of the main lemma and Corollary 6 are given in the next section. A central idea in these proofs is the following elementary observation that is used repeatedly throughout the paper. We begin with some more definitions. A rational circle in $T$ is a set of the form

$$
L=\left\{x+y t: t \in \mathbb{R}^{+}\right\}
$$

for some $x, y=\left(y_{1}, y_{2}\right) \in T$ where both $y_{1}$ and $y_{2}$ are rational and either $y_{1}=1$ or $y_{2}=1$. We will opt for a 'horizontal parameterization' (i.e. $y_{1}=1$ ) whenever possible (in fact, the only circles that we consider that have a vertical parameterization are of the form $L_{j}^{1}$ defined below). Note that any rational circle has finite length as there exists a finite $t$ such that $x+y t=x$. Now, if $L$ intersects $G$ then it does so periodically; let the period of $G$ in $L$ be defined by

$$
p=\min \left\{t \in \mathbb{R}^{+}: \exists g, h \in G \cap L \text { such that } g=h+y t\right\} .
$$

For example, if $g=\left(g_{1}, g_{2}\right) \in G$ and $g_{1} \neq 0$ then the circle generated by $g$, which we define as

$$
L_{g}=\left\{(0,0)+t\left(1, \frac{g_{2}}{g_{1}}\right): t \in \mathbb{R}^{+}\right\},
$$

has period at most $g_{1}$. A second important example of rational circles are the circles

$$
\begin{gathered}
L_{j}^{1}=\left\{\left(j / n_{1}, 0\right)+t(0,1): t \in \mathbb{R}^{+}\right\} \quad \text { for } \quad j=0, \ldots, n_{1}-1 \quad \text { and } \\
L_{j}^{2}=\left\{\left(0, j / n_{2}\right)+t(1,0): t \in \mathbb{R}^{+}\right\} \quad \text { for } \quad j=0, \ldots, n_{2}-1 .
\end{gathered}
$$

For $i \in\{1,2\}$ the circle $L_{j}^{i}$ has period $n_{i} / n$. Our elementary observation is the following: if $L \cap P$ contains a segment longer than the period of $G$ in $L$ (where the length of the segment is measured in terms of the parameterization of $L$ ) then $G$ intersects $P$. To be more precise,

$$
\exists z \in L \text { such that } z+y t \in P \text { for } 0 \leq t \leq p \Rightarrow G \cap P \neq \emptyset .
$$

With this observation in hand, we are ready to apply the main lemma to the group $G$ defined in (7) and parallelogram $P$ described in (8) and (9). To be more precise, we apply the lemma to a large rectangle $R$ contained in $P$. As the dimensions of $P$ depend on $v_{1}$, such a large rectangle will not exist if $v_{1}$ is too large. So, for the sake of this discussion, we assume $v_{1} \leq 1 / 4$. It follows from this assumption that $R=[1 / 6,5 / 6] \times[1 / 3,2 / 3] \subseteq P$. Since $R$ is a rectangle having width $2 / 3$ and height $1 / 3$, Corollary 6 implies that $G$ intersects $P$ unless one of the following conditions hold:

1. $|G|=1,(1 / n, 0) \in G$ or $(0,1 / n) \in G$.

2. $(2 / n, 0) \in G$. 
3. there exists $(u, v) \in G$ such that $u \in\{1 / n, 2 / n\}$ and $v= \pm u$.

4. $(2 / n, 1 / n) \in G$ or $(2 / n,-1 / n) \in G$.

In these cases the condition $(u, v) \in G$ assumes $(u, v)$ is minimal in that there does not exist $\left(u^{\prime}, v^{\prime}\right) \in G$ and integer $k>1$ such that $u=k u^{\prime}$ and $v=k v^{\prime}$.

In the discussion that follows, we show that $G$ and $P$ do, in fact, intersect when conditions 2, 3 or 4 are satisfied. Condition 1, on the other hand, is a completely different matter. The condition $(0,1 / n) \in G$ implies $\left\{v_{3} t_{0}\right\}=\left\{t_{0}\right\}=0$ which means that runner 3 is at 0 whenever runner 3 passes runner 2. So, in this case there is obviously no 3-gap that is also a 23-gap. The condition $(1 / n, 0) \in G$, on the other hand, implies $\left\{v_{1} t_{0}\right\}=0$ which corresponds to runner 1 being at 0 whenever runner 3 passes runner 2 . In such a situation there is clearly no 23-gap that is also a 123-gap. When $|G|=1$ we have $\left\{v_{1} t_{0}\right\}=\left\{v_{3} t_{0}\right\}=0$; in words, when runner 3 passes runner 2 , runners 1,2 and 3 are at 0. Thus, we cannot use Lemma 4 when condition 1 is satisfied: different arguments are required. These arguments are fairly intricate (especially in the case $\left\{v_{1} t_{0}\right\}=0$ ), and are relegated to later sections of the paper. We now return to conditions 2,3 and 4 , dealing with them case by case.

Case 2.1. $(2 / n, 0) \in G$.

Here we must have $n_{2}=2$ and $n \geq 4$. Consider the circle $L_{1}^{2}$. The period of $G$ in $L_{1}^{2}$ is $n_{2} / n=2 / n \leq 1 / 2$ while $L \cap P$ contains a segment of length $2 / 3$. It then follows from (11) that $G \cap P \neq \emptyset$.

Case 2.2. There exists $(u, v) \in G$ such that $u \in\{1 / n, 2 / n\}$ and $v= \pm u$.

If $n=2$ then $(1 / 2,1 / 2) \in G \cap R$. For $n \geq 3$ consider the circle $L$ generated by $(u, v)$. The period of $G$ in $L$ is $u$, and $L \cap P$ contains a segment of length at least $1 / 3$. Thus, if $u \leq 1 / 3$ it follows from (11) that $G \cap P \neq \emptyset$. On the other hand, it follows from $n \geq 3$ that $u \leq 2 / 3$. So, if $u>1 / 3$ then $1 / 3<u \leq 2 / 3$ and $(u, v)$ itself lies in $G \cap P$.

Case 2.3. There exists $v= \pm 1 / n$ such that $(2 / n, v) \in G$.

Consider the circle generated by $(2 / n, v)$. The period of $G$ in $L$ is $2 / n$, and $L \cap P$ contains a segment of length $1 / 3$. Thus, (11) implies $G \cap P \neq \emptyset$ for $n \geq 6$. For $n=3,4,5$ it is easy to see that either $(2 / n, v)$ itself is in $G \cap P$ or $2(2 / n, v) \in G \cap P$.

Note that for $n=4$ the only elements of $G \cap L$ in $P$ lie on the boundary of $P$. Thus, for $v_{1}>1 / 4$ such a group corresponds to a set of speeds for which there exists no 3-gap that is also a 123-gap. This is the fact that motivated our choice of $v_{1} \leq 1 / 4$ for this discussion.

To summarize, we have shown in this section that $\mathcal{B} \neq \mathbb{R}^{+}$under the assumptions $v_{1} \leq 1 / 4,\left\{t_{0}\right\} \neq 0$ and $\left\{v_{1} t_{0}\right\} \neq 0$. As noted above, we handle the cases $\left\{t_{0}\right\}=0$ and $\left\{v_{1} t_{0}\right\}=0$ via applications of the main lemma. Each of these applications, like the application given in this section, require that we assume $v_{1}$ is small, and become easier 
as the assumed value of $v_{1}$ shrinks. We handle large $v_{1}$ using different ad hoc arguments, and as $v_{1}$ shrinks these arguments become more difficult. Thus, we have a tradeoff in the difficulty of the proof depending on where we 'switch' between an ad hoc proof and

applications of the main lemma. In this paper, we make the transition at $v_{1}=1 / 3$, but this choice is arbitrary.

The rest of this paper is organized as follows. The next section, Section 3, contains the proofs of the main lemma and Corollary 6 as well as an additional technical lemma that will be used in all later applications of the main lemma. In Section 4 the case of irrational speeds is considered. In Section 5 we handle the case $\left\{v_{1} t_{0}\right\}=0$, and in Section 6 we deal with $\left\{t_{0}\right\}=0$. In both Section 5 and Section 6 we assume $v_{1}<1 / 3$. In Section 7 we consider the case $\left\{t_{0}\right\} \neq 0,\left\{v_{1} t_{0}\right\} \neq 0$ and $1 / 4<v_{1}<1 / 3$; this is merely an extension of the argument in this section to slightly larger values of $v_{1}$. In Section 8 we consider $v_{1} \geq 1 / 3$.

Taken together, these sections constitute a proof of Theorem 2. A proof of Theorem 3 is obtained by following that of Theorem 2 and observing that, except for the sets of speeds specified in Theorem 3, the argument provides an uncovered interval, or may be easily enhanced so that it will. We omit the extra details needed for that.

\section{Tools}

\subsection{Proof of the Main Lemma}

Let $G$ be a finite subgroup of $T$ of order $n>1$, let $0<\alpha, \beta<1$ be given such that $\alpha \beta \geq 1 / n$, and let $K$ be an arbitrary symmetric closed convex neighborhood of $(0,0)$ in $\mathbb{R}^{2}$ which is contained in the box $[-\alpha n, \alpha n] \times[-\beta n, \beta n]$ and has area $A(K) \geq 4 n$.

We first show that there exists an element $(i, j) \in\left(\mathbb{Z}^{2} \cap K\right) \backslash\{(0,0)\}$ such that $(i / n, j / n) \in G$ (this statement could be deduced from a well-known theorem of Minkowski, but we prefer to give a proof here). Consider the family $\mathcal{F}$ of subsets of $T$ of the form $(i / n, j / n)+(1 / 2 n) K$, where $(i / n, j / n) \in G$. If two sets in $\mathcal{F}$ intersect, say $\left(i_{1} / n, j_{1} / n\right)+$ $1 / 2 n\left(x_{1}, y_{1}\right)=\left(i_{2} / n, j_{2} / n\right)+1 / 2 n\left(x_{2}, y_{2}\right)$ for $\left(x_{1}, y_{1}\right),\left(x_{2}, y_{2}\right) \in K$, then, $\left(i_{1}-i_{2}, j_{1}-j_{2}\right)=$ $1 / 2\left(x_{2}, y_{2}\right)+1 / 2\left(-x_{1},-y_{1}\right)$ holds modulo $n$, and we obtain an element as desired. But it is impossible for the sets in $\mathcal{F}$ to be disjoint, as there are $n$ of them and each is a closed set of area at least $1 / n$, with $n>1$.

We now pick an $(i, j)$ as above which is minimal in the sense that it is not of the form $(i, j)=k\left(i_{0}, j_{0}\right)$ for some integer $k>1$ and $\left(i_{0} / n, j_{0} / n\right) \in G$. We will show that if $G$ misses some $\alpha \times \beta$ rectangle $R$ in $T$ then $(i, j)$ must satisfy

$$
\beta|i|+\alpha|j|-\frac{2}{n}|i||j|<1
$$

This will establish the condition stated in part 2 of the lemma. In order to obtain the condition stated in part 1 of the lemma, it suffices to take $K$ to be the box $[-1 / \beta, 1 / \beta] \times$ $[-\beta n, \beta n]$ and to observe that $\alpha \beta \geq 2 / n,(i, j) \in K$ and (12) imply $-1 / \beta<i<1 / \beta$, $-1 / \alpha<j<1 / \alpha$. 
We assume w.l.o.g. that $i, j \geq 0$. If $i=0$ then $G$ consists of $n / j$ equidistant points on each of the $j$ vertical circles $L_{0}^{1}, \ldots, L_{j-1}^{1}$. Since the distance between two consecutive points on one of these vertical circles is $j / n \leq \beta$ (recall, $K \subseteq[-\alpha n, \alpha n] \times[-\beta n, \beta n]$ ), in order for $G$ to miss $R$ the latter must lie in the interior of a strip between two vertical circles. But, the distance between two vertical circles is $1 / j$ which is no more than $\alpha$, unless (12) holds. The argument in the case $j=0$ is similar.

Thus, we may assume $i, j>0$. The circle $L_{g}$ generated by $g=(i / n, j / n)$ has period (measured horizontally) exactly $i / n$ (due to the minimality of $(i, j)$ ). Consider the family $\mathcal{L}$ of line segments which are parallel to $L_{g}$, pass through a point of $G$ and have the same projection onto the horizontal axis as $R$. By (11), if the intersection of such a line segment with $R$ has length (measured horizontally) at least $i / n$, then $G \cap R \neq \emptyset$. Let $\left(x_{1}, x_{2}\right)$ be the lower left hand corner of $R$. Since $i / n \leq \alpha$ and $j / n \leq \beta$ (which is due to the fact that $K \subseteq[-\alpha n, \alpha n] \times[-\beta n, \beta n])$, the intersection of a line in $\mathcal{L}$ with $R$ is of length at least $i / n$ if and only if the line intersects the set

$$
\left\{x_{1}+\frac{i}{n}\right\} \times\left[x_{2}+\frac{j(2 i-\alpha n)}{i n}, x_{2}+\beta\right] .
$$

The number of line segments in $\mathcal{L}$ is determined by

$$
|\mathcal{L}|=\left|G \cap\left(\left[x_{1}, x_{1}+i / n\right) \times[0,1)\right)\right|=i .
$$

Since $G$ is a group, these line segments are equally spaced. Hence, these line segments cross the vertical circle $\left\{x_{1}+\frac{i}{n}\right\} \times[0,1)$ at equally distanced points, and if none of these has second coordinate between $x_{2}+\frac{j(2 i-\alpha n)}{i n}$ and $x_{2}+\beta$, then we must have

$$
\beta-\frac{j(2 i-\alpha n)}{i n}<\frac{1}{i}
$$

or equivalently (12).

\subsection{Proof of Corollary 6}

Let $G$ be a finite subgroup of $T$ of order $n>1$, let $0<\alpha, \beta<1$ and define

$$
X=\left\{(i, j) \in \mathbb{Z}^{2}: 0 \leq i<\frac{1}{\beta},-\frac{1}{\alpha}-\frac{\beta}{\alpha} i<j<\frac{1}{\alpha}+\frac{\beta}{\alpha} i\right\} \backslash\{(0,0)\} .
$$

We show that either $G$ intersects every $\alpha \times \beta$ rectangle in $T$ or there exists $(i / n, j / n) \in G$ such that $(i, j) \in X$. If $n \geq 2 / \alpha \beta$ this follows directly from part 1 of the Main Lemma.

Assume $1 / \beta \leq n<2 / \alpha \beta$. Define

$$
K=\left\{(x, y) \in \mathbb{R}^{2}:-\frac{1}{\beta}<x<\frac{1}{\beta},-\beta n \leq y \leq \beta n\right\} .
$$

As in the proof of the Main Lemma, there exists a minimal $(i, j) \in\left(\mathbb{Z}^{2} \cap K\right) \backslash\{(0,0)\}$ such that $i \geq 0$ and $(i / n, j / n) \in G$ (note that the conditions $n \geq 1 / \beta$ and $\beta<1$ guarantee 
that $(1 / 2 n) K$ does not self-intersect on the torus, and that although it is only half-closed $n$ translates of it cannot be disjoint). If $n<1 / \alpha \beta$ then $(i, j) \in X$, and the proof is complete. If $1 / \alpha \beta \leq n<2 / \alpha \beta$ then either $(i, j) \in X$ or we have

$$
|j| \geq \frac{1}{\alpha}+\frac{\beta i}{\alpha} \quad \text { and } \quad|j| \leq \beta n .
$$

But, if the latter holds then we obtain

$$
\begin{aligned}
\beta|i|+\alpha|j|-\frac{2|i||j|}{n} & \geq \beta i+\alpha\left(\frac{1}{\alpha}+\frac{\beta i}{\alpha}\right)-\frac{2 i|j|}{n} \\
& =1+2 i\left(\beta-\frac{|j|}{n}\right) \\
& \geq 1+2 i\left(\beta-\frac{\beta n}{n}\right) \\
& =1
\end{aligned}
$$

and it follows from the proof of the main lemma that $G$ intersects all $\alpha \times \beta$ rectangles.

Finally, assume $n<1 / \beta$. Then, since $\alpha<1, X$ contains the set

$$
\{0,1, \ldots, n-1\} \times\{-1,0,1\} \backslash\{(0,0)\},
$$

which must contain some $(i, j)$ such that $(i / n, j / n) \in G$.

\subsection{Minimal group elements}

In the applications of the main lemma in the following sections there are many pairs $(i, j)$ that satisfy (10) (i.e. $\alpha$ and $\beta$ are small). In these situations there are groups of small order that contain more than one element that satisfies (10). Thus, when we consider the pairs $(i, j)$ that satisfy (10) case by case, these particular small groups could be considered more than once.

In order to attempt to avoid this repetition, we strengthen the notion of minimality used in the proofs of the Main Lemma and Corollary 6. For $i, j \geq 0$ let $Z_{i, j}$ be the following subset of $\mathbb{Z}^{2}$

$$
Z_{i, j}=[-i, i] \times[-j, j] \backslash\{( \pm i, \pm j)\} .
$$

We say that $(i / n, j / n) \in G$ is minimal if the following holds:

$$
\left\{(k / n, l / n):(k, l) \in Z_{|i|,|j|}\right\} \cap G=\{(0,0)\} .
$$

Note that, in spite of our terminology, minimality is a property of the pair $(i, j)$ and not of the group element $(i / n, j / n)$; in other words, it may happen that $(i / n, j / n),\left(i^{\prime} / n, j^{\prime} / n\right)$ represent the same group element and one is minimal while the other is not.

We have the following lower bound on the order of a group having minimal element $(i / n, j / n)$. 
Lemma 7. Let $G$ be a group of order $n>1$ having minimal element $(i / n, j / n)$. Then we have

$$
\begin{gathered}
|i|,|j| \geq 2 \quad \Rightarrow \quad n \geq(|i|+1)(|j|+1)-1 \\
|i|,|j| \geq 1 \quad \Rightarrow \quad n \geq(|i|+1)(|j|+1)-2 \\
i=0 \text { or } j=0 \quad \Rightarrow \quad n \geq 2 \max \{|i|,|j|\} .
\end{gathered}
$$

Proof. Suppose $i, j \neq 0$. Let $Y$ be the following subset of $\mathbb{Z}^{2}$

$$
Y=[0,|i|] \times[0,|j|] \backslash\{(|i|, 0),(|i|,|j|)\}
$$

Note that $Y-Y \subseteq Z_{|i|,|j|}$. We will show that for $\left(a_{1}, a_{2}\right) \neq\left(b_{1}, b_{2}\right)$ we have

$$
\left(a_{1} / n, a_{2} / n\right),\left(b_{1} / n, b_{2} / n\right) \in G \Rightarrow\left(\left(a_{1}, a_{2}\right)+Y\right) \cap\left(\left(b_{1}, b_{2}\right)+Y\right)=\emptyset
$$

(where addition is modulo $n$ ). If (15) holds then $\{g+Y / n: g \in G\}$ (where addition is in $T)$ is a collection of disjoint subsets of $\{(k / n, l / n): 0 \leq k, l<n\}$. Thus, $|G||Y| \leq n^{2}$, and the second part of (14) follows. The first part of (14) follows from the observation that when $|i|,|j| \geq 2$ the shape $Y / n$ cannot tile the torus.

It remains to prove (15). Assume for the sake of contradiction that $a=\left(a_{1} / n, a_{2} / n\right), b=$ $\left(b_{1} / n, b_{2} / n\right) \in G,\left(x_{1}, x_{2}\right),\left(y_{1}, y_{2}\right) \in Y$ and $\left(a_{1}, a_{2}\right)+\left(x_{1}, x_{2}\right)=\left(b_{1}, b_{2}\right)+\left(y_{1}, y_{2}\right)$. It follows that $a-b=\left(y_{1} / n-x_{1} / n, y_{2} / n-x_{2} / n\right) \in G$. However, $\left(y_{1}-x_{1}, y_{2}-x_{2}\right) \in Z_{|i|,|j|}$. This contradicts the minimality of $(i / n, j / n)$.

If $i=0$ or $j=0$ the conclusion of (14) is straightforward.

Now, in the proofs of the Main Lemma and Corollary 6 , we chose $(i, j) \in\left(\mathbb{Z}^{2} \cap K\right) \backslash$ $\{(0,0)\}$ such that $(i / n, j / n) \in G, i \geq 0$ and $(i, j)$ is not a positive integer multiple of $\left(i_{0}, j_{0}\right) \in \mathbb{Z}^{2}$ such that $\left(i_{0} / n . j_{0} / n\right) \in G$. Note that in both proofs we can actually choose an element $\left(i^{\prime}, j^{\prime}\right) \in\left(\mathbb{Z}^{2} \cap K\right) \backslash\{(0,0)\}$ such that $i^{\prime} \geq 0$ and $\left(i^{\prime} / n, j^{\prime} / n\right)$ is a minimal element of $G$ in the place of $(i, j)$. We thereby conclude that either $G$ intersects every $\alpha$ by $\beta$ rectangle or there exists $(i, j) \in \mathbb{Z}^{2} \backslash\{(0,0)\}$ satisfying (10) and (14).

\section{Irrational Speeds}

In this section we justify why, in the rest of this paper, we restrict our attention to rational speeds. In some of the previous papers that treated Conjecture 1, a reduction of the irrational case to the rational case was attributed to Wills. However, it seems to us that what Wills did does not amount to that, and therefore we address the issue here.

It follows from the lemma below that if Conjecture 1 holds when $n-1$ is substituted for $n$ and all speeds are assumed to be rational, then it also holds true (in fact, with some slack) for $n$, except possibly when the speeds are proportional to a collection of rational speeds. To see this, apply Lemma 8 with any $\delta$ satisfying $1 / n<\delta<1 /(n-1)$. In particular, the irrational case of Conjecture 1 for $n=6$ follows from the rational case of the conjecture for $n=5$ (proved in [CP] and [BGGST]). Similarly, the irrational case of Conjecture 1 for $n=7$ is implied by the results of the current paper. 
Lemma 8. Let $0<\delta<1 / 2$. Suppose that for every collection $v_{1}, \ldots, v_{n-2} \in \mathbb{Q}^{+}$there exists $t \in \mathbb{R}^{+}$satisfying

$$
\left\{v_{i} t\right\} \in(\delta, 1-\delta) \text { for } i=1, \ldots, n-2 .
$$

Then for any collection $u_{1}, \ldots, u_{n-1} \in \mathbb{R}^{+}$for which there is a pair $u_{i}, u_{j}$ such that $u_{i} / u_{j} \notin \mathbb{Q}$ there exists $t \in \mathbb{R}^{+}$satisfying

$$
\left\{u_{i} t\right\} \in(\delta, 1-\delta) \text { for } i=1, \ldots, n-1 \text {. }
$$

Proof. Let $\mathbf{u}=\left(u_{1}, \ldots, u_{n-1}\right)$ and consider the set

$$
M(\mathbf{u})=\left\{\mathbf{y} \in \mathbb{R}^{n-1}: \exists t \in \mathbb{R} \text { and } \mathbf{k} \in \mathbb{Z}^{n-1} \text { such that } \mathbf{y}=t \mathbf{u}-\mathbf{k}\right\} .
$$

Clearly, the conclusion of the lemma is equivalent to the assertion that $M(\mathbf{u})$ intersects the open hypercube $(\delta, 1-\delta)^{n-1}$. It suffices to prove that the closure of $M(\mathbf{u})$, which we denote by $\bar{M}(\mathbf{u})$, intersects the same hypercube.

By a generalization of Kronecker's theorem (see [P, Satz 65]), the set $\bar{M}(\mathbf{u})$ is characterized as follows. If $u_{1}, \ldots, u_{n-1}$ are linearly independent over $\mathbb{Q}$, then $\bar{M}(\mathbf{u})=\mathbb{R}^{n-1}$; in this case there is nothing to prove. Otherwise, the speeds $u_{1}, \ldots, u_{n-1}$ satisfy some homogeneous linear equations over $\mathbb{Q}$ of the form

$$
a_{1} u_{1}+\cdots+a_{n-1} u_{n-1}=0 .
$$

Consider a maximal set of linearly independent equations of the form (16) satisfied by $u_{1}, \ldots, u_{n-1}$, and write them as the rows of a matrix $A$; thus, $A$ is a $m \times(n-1)$ matrix over $\mathbb{Q}$ of rank $m$, satisfying $\mathbf{u} \in \operatorname{Ker}(A)$. In this setting, the above mentioned generalization of Kronecker's theorem is the statement

$$
\bar{M}(\mathbf{u})=\operatorname{Ker}(A)+\mathbb{Z}^{n-1} .
$$

Since $\operatorname{Ker}(A)$ contains the vector $\mathbf{u}$ which lies in the positive orthant of $\mathbb{R}^{n-1}$, and $A$ has rational entries, it follows that $\operatorname{Ker}(A)$ also contains some vector $\mathbf{r}$ with positive rational components. By assumption, $\mathbf{u}$ is not proportional to a rational vector, and hence $\operatorname{Ker}(A)$ has dimension two or more. We can therefore find a vector $\mathbf{s} \in \operatorname{Ker}(A)$ which has rational components and is not a constant multiple of $\mathbf{r}$. It follows that we can find $i, j$ obeying

$$
\frac{s_{i}}{r_{i}}<\frac{s_{j}}{r_{j}} \text { and } \frac{s_{k}}{r_{k}} \notin\left(\frac{s_{i}}{r_{i}}, \frac{s_{j}}{r_{j}}\right) \text { for } k=1, \ldots, n-1 .
$$

Consider now the vector

$$
\mathbf{w}=\left(r_{i}+r_{j}\right) \mathbf{s}-\left(s_{i}+s_{j}\right) \mathbf{r} .
$$

It is clearly a rational vector in $\operatorname{Ker}(A)$. We observe that we have

$$
w_{i}=-w_{j} \text { and } w_{k} \neq 0 \text { for } k=1, \ldots, n-1 \text {. }
$$

THE ELECTRONiC JOURNAL of COMBINATORICS 8 (NO. 2) (2001), \#R3 
To verify the second part of (19), note that $w_{k}=0$ is equivalent to

$$
\frac{s_{k}}{r_{k}}=\frac{s_{i}+s_{j}}{r_{i}+r_{j}}
$$

which would imply that $s_{k} / r_{k}$ lies between $s_{i} / r_{i}$ and $s_{j} / r_{j}$, contradicting (18).

Now, let $v_{1}, \ldots, v_{n-2}$ be a collection of positive rationals that contains $\left|w_{1}\right|, \ldots,\left|w_{n-1}\right|$; we can find such a collection by virtue of (19). Applying the assumption of the lemma, we find $t \in \mathbb{R}^{+}$such that $\left\{v_{i} t\right\} \in(\delta, 1-\delta)$ for $i=1, \ldots, n-2$. Since $t \mathbf{w} \in \operatorname{Ker}(A)$, it follows from $(17)$ that $\bar{M}(\mathbf{u})$ intersects the hypercube $(\delta, 1-\delta)^{n-1}$, which completes the proof.

\section{$5 \quad\left\{v_{1} t_{0}\right\}=0$ and $x_{1}>3$}

In this case runner 1 is at 0 whenever runner 3 passes runner 2 . In terms of the speeds of the runners, this condition is equivalent to

$$
\frac{1}{1-v_{2}} v_{1}=k \Rightarrow v_{2}=1-\frac{v_{1}}{k}
$$

for some integer $k \geq 1$. As we have already noted, there is no chance of applying Lemma 4 when (20) holds (i.e. when (20) holds there exists no 123-gap of length $2 / 3$ ). The difficulty is further compounded by the fact that the behavior of the system varies depending on the value of $k$ in (20). In order to deal with these differences, we divide our treatment of this topic into two cases.

Case 5.1. $t_{0} v_{1}=k$ for some integer $k \geq 3$.

While there exists no 123-gap of length $2 / 3$, we will show that in this situation there usually are two (or more) 123-gaps of length at least $1 / 2$ near each other. To make this notion more precise, we establish some definitions. For a fixed time $t$ define

$$
I_{1}=[t, t+1 / 2], I_{2}=(t+1 / 2, t+1) \text { and } I_{3}=[t+1, t+3 / 2] .
$$

We say that $t$ initiates the triple configuration $I_{1}, I_{2}, I_{3}$ if

$$
I_{1} \cap\left(B_{1} \cup B_{2} \cup B_{3}\right)=\emptyset \text { and } I_{3} \cap\left(B_{1} \cup B_{2} \cup B_{3}\right)=\emptyset \text {. }
$$

Before analyzing if and when triple configurations arise, we show that $x_{4}$ and $x_{5}$ must satisfy very special conditions if we are to have

$$
I_{1} \cup I_{3} \subseteq B_{4} \cup B_{5}
$$

For the purposes of this discussion, we assume $t$ initiates a triple configuration and that (21) holds. Our first observation is that Lemma 4 implies

$$
x_{4}>3 / 4 \text {. }
$$

THE ELECTRONiC JOURNAl OF COMBinatorics 8 (NO. 2) (2001), \#R3 
Claim 9. If $I_{1} \cup I_{3} \subseteq B_{4} \cup B_{5}$ then no 4-block is contained in $I_{2}$.

Proof. Assume for the sake of contradiction that there exists $j$ satisfying

$$
(j-1 / 6) x_{4},(j+1 / 6) x_{4} \in I_{2} .
$$

This implies

$$
\left[(j-5 / 6) x_{4}, t+1 / 2\right],\left[t+1,(j+5 / 6) x_{4}\right] \subseteq B_{5},
$$

and therefore we have

$$
\begin{aligned}
2 x_{4} / 3 & >2 x_{5} / 3 \\
& >(t+1 / 2)-(j-5 / 6) x_{4}+(j+5 / 6) x_{4}-(t+1) \\
& =5 x_{4} / 3-1 / 2 .
\end{aligned}
$$

Therefore, $x_{4}<1 / 2$, which contradicts (22).

Claim 10. If $I_{1} \cup I_{3} \subseteq B_{4} \cup B_{5}$ then no 4-block is contained in $I_{1}$ or $I_{3}$.

Proof. Assume without loss of generality that there exists $j$ satisfying

$$
t \leq(j-1 / 6) x_{4} \text { and }(j+1 / 6) x_{4} \leq t+1 / 2 .
$$

This implies

$$
2 x_{5} / 3<x_{4} / 3 \text { and } 2 x_{5} / 3+x_{4} / 3>1 / 2,
$$

and therefore $1 / 4<x_{5}<1 / 2$. This absolute upper bound, in turn, implies $x_{4} / 3+x_{5} / 3<$ $1 / 2$. Thus, we must have

$$
t+1 \leq(j+5 / 6) x_{4} \text { and }(j+7 / 6) x_{4} \leq t+3 / 2 .
$$

Now, (23) and (25) imply that there exist four 1234-gaps in $I_{1} \cup I_{3}$ that are covered by $B_{5}$. It follows from this observation and $1 / 4<x_{5}$ that no 5 -block is contained in the 4-block $\left((j-1 / 6) x_{4},(j+1 / 6) x_{4}\right)$. So, the first two 1234-gaps in $I_{1} \cup I_{3}$ must be covered by consecutive 5 -blocks, which implies

$$
4 x_{5} / 3>1 / 2 .
$$

From this observation it follows that no 5-block is contained in $I_{2}$, and therefore the first three 1234-gaps in $I_{1} \cup I_{3}$ must be covered by consecutive 5 -blocks. We therefore have $4 x_{5} / 3>2 x_{4} / 3$, which contradicts $(24)$.

To summarize, we have shown that if $t$ initiates a triple configuration and (21) holds then there exists a $j$ such that the following holds:

$$
\begin{array}{cc}
\text { either } & {\left[(j-1 / 6) x_{4}<t \text { and }(j+3 / 2) x_{4}>t+3 / 2\right]} \\
\text { or } & {\left[(j-1 / 2) x_{4}<t \text { and }(j+7 / 6) x_{4}>t+3 / 2\right] .}
\end{array}
$$


One implication of $(26)$ is

$$
10 x_{4} / 6>3 / 2 \Rightarrow x_{4}>9 / 10 .
$$

It also follows from (26) that if there exists $t$ satisfying

$$
([t, t+1 / 2] \cup[t+1, t+3 / 2] \cup \ldots \cup[t+l, t+l+1 / 2]) \cap\left(B_{1} \cup B_{2} \cup B_{3}\right)=\emptyset
$$

(we call this a multiple configuration) then there exists a $j$ for which we have:

$$
\begin{gathered}
\text { either } \quad(j-1 / 6) x_{4}<t \text { and }(j+l+1 / 2) x_{4}>t+l+1 / 2, \\
\text { or } \quad(j-1 / 2) x_{4}<t \text { and }(j+l+1 / 6) x_{4}>t+l+1 / 2 .
\end{gathered}
$$

This implies

$$
(l+2 / 3) x_{4}>l+1 / 2 \Rightarrow x_{4}>\frac{6 l+3}{6 l+4} .
$$

We now turn to an analysis of how triple and multiple configurations arise. Our first observation here is the following: if $t$ satisfies

$$
j k x_{1} \leq t \leq(j+1 / 6) k x_{1}
$$

then runners 2 and 3 are very close to each other. In fact, if (31) holds then (20) implies

$$
\left\{\left\{v_{3} t\right\}-\left\{v_{2} t\right\}\right\}=\left\{t-\left(1-\frac{v_{1}}{k}\right) t\right\}=\left\{t \frac{v_{1}}{k}\right\} \leq 1 / 6 .
$$

Thus, if $J$ is a 23-gap and $J \subseteq\left[j k x_{1},(j+1 / 6) k x_{1}\right]$ then $|J| \geq 1 / 2$. More importantly, if we have

$$
x_{1} / 6 \leq i+1 / 3, i+l+1 / 3 \leq k x_{1} / 6 \text { and } i+l+5 / 6 \leq 5 x_{1} / 6
$$

then $t=i+1 / 3$ and $l$ satisfy (28). It then follows from (29) that we must have

$$
(i+1 / 2) x_{4}<i+1 / 3 \Rightarrow x_{4}<\frac{6 i+2}{6 i+3} .
$$

It follows from (30) and (33) that we have a contradiction if $l \geq i$ (note that our multiple configuration begins in the 123-gap following the time $i$ and ends in the 123-gap following time $i+l$ ). A multiple configuration satisfying $l \geq i$ exists unless one of the following conditions holds.

1. $k=3$ and $x_{1}<14 / 3$

2. $k=3$ and $8<x_{1}<26 / 3$

3. $k=4$ and $x_{1}<14 / 4$

4. $k \geq 5$ and $x_{1}<17 / 5$.

We give a unified argument for three of these conditions.

Case 5.1.1. $k \geq 3$ and $x_{1}<14 / 3$. 
Since $v_{1}<1 / 3$ and $k \geq 3$, we have

$$
v_{2}=1-\frac{v_{1}}{k}>1-\frac{1}{3 \cdot 3}=\frac{8}{9}
$$

Therefore, $[21 / 16,11 / 6]$ is contained in a 123-gap, implying

$$
\frac{2 x_{4}}{3}>\frac{11}{6}-\frac{21}{16}=\frac{25}{48} \text {. }
$$

It follows that $[21 / 16,275 / 192]$ is contained in a 1234-gap. So, there exists an $i \geq 2$ such that $(i-1 / 6) x_{5}<21 / 16$ and $275 / 192<(i+1 / 6) x_{5}$. The only values of $i$ that may satisfy these equations are $i=2,3$. Thus, we obtain

$$
19 x_{5}>11 x_{4} \Rightarrow x_{5}>x_{4} / 2
$$

In particular, if $13 x_{4} \leq 11$ then $\left[13 x_{4} / 6,11 / 6\right]$ is a 1234 -gap that cannot be covered by runner 5 . So, we may assume $13 x_{4}>11$. But this implies that $[21 / 16,121 / 78]$ is a 1234 gap. For runner 5 to cover this gap, there must be $i \in\{2,3\}$ such that $(i-1 / 6) x_{5}<21 / 16$ and $121 / 78<(i+1 / 6) x_{5}$. However (by the narrowest of margins) no such $i$ exists.

Case 5.1.2. $k=3$ and $8<x_{1}<26 / 3$.

In this situation, there exists a triple configuration $((28)$ holds with $t=14 / 6$ and $l=1$ ). It then follows from $(27)$ that $x_{4}>9 / 10$. Furthermore, we have

$$
v_{2}=1-\frac{v_{1}}{3}>1-\frac{1}{24}=\frac{23}{24} .
$$

It follows from these two observations that $[52 / 23,51 / 20]$ is contained in a 1234-gap. Thus, there exists an $i \geq 3$ such that $(i-1 / 6) x_{5}<52 / 23$ and $(i+1 / 6) x_{5}>51 / 20$. No such $i$ exists.

Case 5.2. $v_{1} t_{0}=k$ for $k \in\{1,2\}$.

In this case the above method of exploiting triple configurations will not work. We shall handle the cases $k=1,2$ jointly by proving the following

Proposition 11. Let $v_{1}<v_{2}<v_{3}<v_{4}<v_{5}$ be a collection of rational speeds satisfying $v_{1}+v_{2}=v_{3}=1$.

1. If $v_{1}<1 / 3$ then there exists a positive real $t^{*}$ such that $\left\{v_{i} t^{*}\right\} \in[1 / 6,5 / 6]$ for $i=1, \ldots, 5$.

2. If, moreover, $v_{1}<1 / 6$, then there exists $t^{*}$ satisfying the requirements in part 1 as well as $\left\{v_{1} t^{*}\right\} \notin(5 / 12,7 / 12)$. 
In the case $k=1,(20)$ becomes $v_{1}+v_{2}=1$, and we apply part 1 of the proposition to conclude that $\mathcal{B} \neq \mathbb{R}^{+}$. In the case $k=2$, (20) becomes $v_{1} / 2+v_{2}=1$. By applying part 2 of the proposition to the collection of speeds $v_{1} / 2, v_{2}, v_{3}, v_{4}, v_{5}$, we obtain $t^{*}$ such that $\left\{v_{i} t^{*}\right\} \in[1 / 6,5 / 6]$ for $i=2, \ldots, 5$ and $\left\{\left(v_{1} / 2\right) t^{*}\right\} \in[1 / 6,5 / 6] \backslash(5 / 12,7 / 12)$. The latter implies that $\left\{v_{1} t^{*}\right\} \in[1 / 6,5 / 6]$, so that $\mathcal{B} \neq \mathbb{R}^{+}$for the original collection of speeds.

The remainder of this section is devoted to the (long) proof of Proposition 11, which is organized into the following parts. We begin in Part 1 by making some initial assumptions and fixing notation. Part 2 consists of a number of preliminary developments based on the initial assumptions. These include showing that $v_{4}$ is small, showing that the denominators of $v_{4}$ and $v_{5}$ equal the denominator of $v_{1}$, and eliminating the $v_{1}=1 / 4$ case. With these observations in hand we are able to apply the main lemma in Part 3. Before handling the (many) exceptional groups that are left by this application of the main lemma we develop in Part 4 techniques for getting an expression for $v_{4}$ in terms of $v_{1}$ for each exceptional group. These expressions are then used in Part 5 to show that all exceptional groups but one actually intersect the polygon defined in Part 3. The remaining exceptional group (which does not necessarily intersect the polygon defined in Part 3) is treated (using different techniques) in Part 6. This organization is presented schematically in Figure 2.

We begin with our initial assumptions and notation. We proceed indirectly in a way that proves part 1 and 2 of the Proposition simultaneously. Define:

$$
\mathcal{B}^{\prime}= \begin{cases}\mathcal{B} \cup 1 / 2 B_{1} & \text { if } v_{1}<1 / 6 \\ \mathcal{B} & \text { if } 1 / 6 \leq v_{1}<1 / 3\end{cases}
$$

We assume at the outset that $\mathcal{B}^{\prime}=\mathbb{R}^{+}$. If $v_{1}<1 / 6$ we will, for ease of notation, introduce a fictional runner 0 having speed $2 v_{1}$. This runner is in the interval $(-1 / 6,1 / 6)$ whenever runner 1 is in $(5 / 12,7 / 12)$. This will allow us, for example, to distinguish those 123-gaps during which runner 1 is known not to be in $(5 / 12,7 / 12)$, now 0123-gaps, from 123-gaps in which runner 1 might intersect (5/12,7/12), simply 123-gaps. Note that the times covered by this fictional runner 0 are simply $B_{0}:=1 / 2 B_{1}$. For $1 / 6 \leq v_{1}<1 / 3$ this fictional runner is not introduced. However, the 0 is not dropped from the notation in this setting; for example, we will discuss both 0123-gaps and 123-gaps, but these objects are identical, by convention, for $1 / 6 \leq v_{1}<1 / 3$.

We assume $p$ and $q$ are relatively prime positive integers that satisfy

$$
v_{1}=p / q<1 / 3 \text { and } v_{2}=(q-p) / q \text {. }
$$

We are now ready for Part 2 of the proof of Proposition 11: preliminary observations that will allow us to make some simplifying assumptions on the values of the speeds in the main part of the argument.

Lemma 12. Assume $v_{1}=p / q, v_{2}=(q-p) / q$ and $\mathcal{B}^{\prime}=\mathbb{R}^{+}$. If $I$ is a 0123-gap then $I \subseteq B_{4}$ or $I \subseteq B_{5}$ or there exist integers $r, s$ such that $v_{4}=r / q$ and $v_{5}=s / q$. 


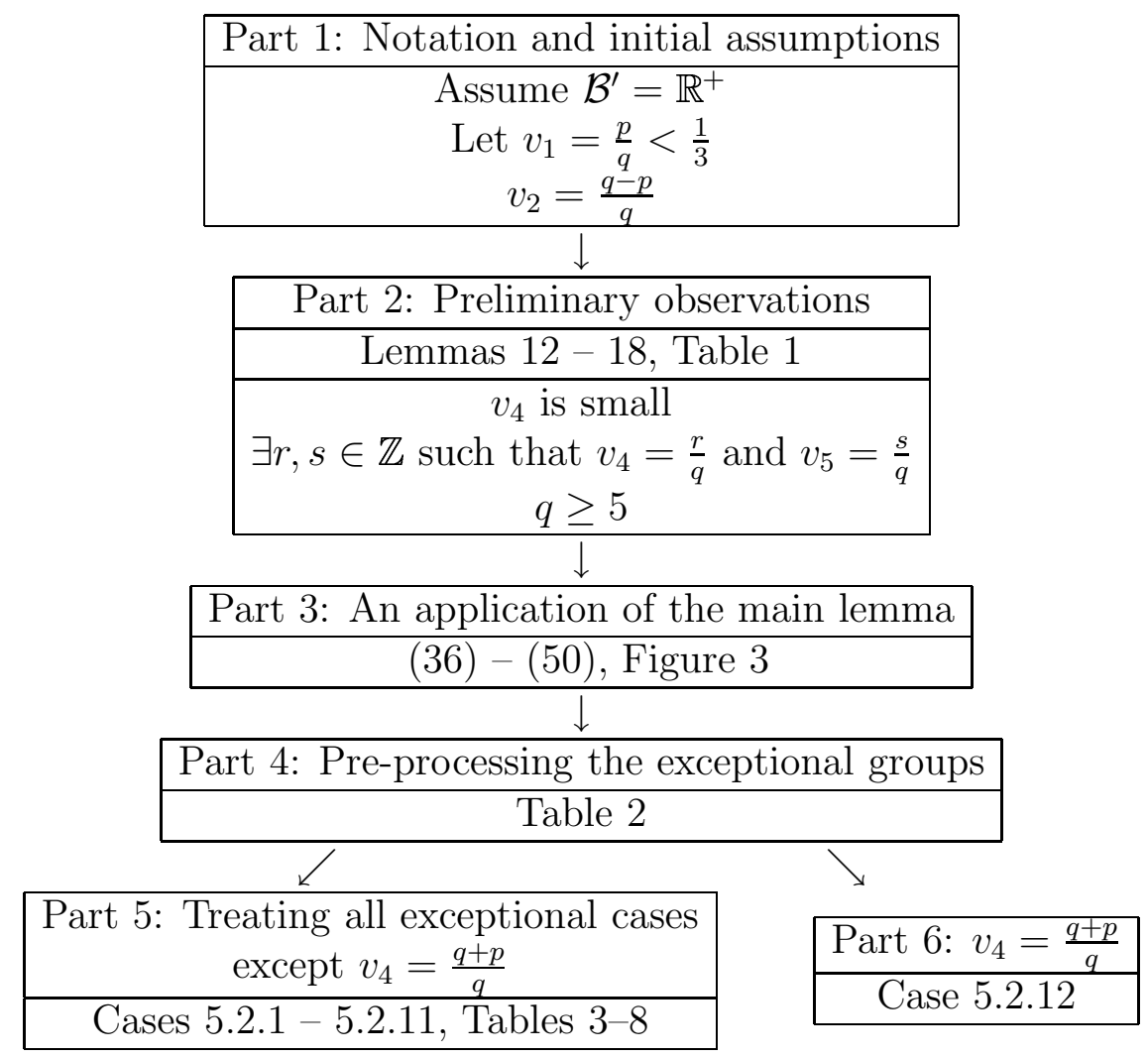

Figure 2: A road-map of the proof of Proposition 11. 
Proof. Suppose $I \nsubseteq B_{4}$ and $I \nsubseteq B_{5}$. Note that $\left\{v_{j} q\right\}=0$ for $j=0,1,2,3$. Thus $I+i q$ is a 0123-gap for $i=0,1, \ldots$ Since $I \subseteq B_{4} \cup B_{5}$, there exists a time $u \in I \cap B_{4} \cap B_{5}$. Furthermore, the time $u+i q$ must be contained in $B_{4} \cup B_{5}$ for $i=1,2, \ldots$ Now, for $j \in\{4,5\}$, the set

$$
P_{j}:=\left\{\left\{v_{j}(u+i q)\right\}: i=0,1 \ldots\right\}
$$

is a finite set that is equidistributed on the circle $[0,1)$. Thus, if $n_{j}=\left|P_{j}\right|$, then we have

$$
\left|\left\{x \in P_{j}: x \notin[1 / 6,5 / 6]\right\}\right| \leq\left\lfloor\frac{n_{j}+2}{3}\right\rfloor .
$$

In particular, if $n_{j}>1$ then the proportion of elements of $P_{j}$ that lie in $(-1 / 6,1 / 6)$ is at most $1 / 2$. Since runners 4 and 5 both cover $u$ itself, it follows from (34) that there exists $a \in\{4,5\}$ such that $n_{a}=1$. It follows that the denominator of $v_{a}$ is $q$.

Let $b$ be the element of $\{4,5\}$ not equal to $a$. Since $I$ is contained in neither $B_{4}$ nor $B_{5}$, there is a $0123 a$-gap $J \subseteq I$. Let $w \in J$. Since $\left\{v_{j} q\right\}=0$ for $j=0,1,2,3, a$, runner $b$ must cover the time $w+i q$ for $i=0,1, \ldots$ However, the set of positions of runner $b$ at these times is equidistributed in $[0,1)$. Therefore, as in (34), there can be only one such position, and the denominator of $v_{b}$ is $q$.

We will find it useful to consider the case $q=4$ separately.

Lemma 13. If $v_{1}=p / q, v_{2}=(q-p) / q$ and $\mathcal{B}^{\prime}=\mathbb{R}^{+}$then $q \geq 5$.

Proof. Suppose $q=4$. Since $v_{1}<1 / 3$, we must have $p=1$. Thus, $v_{1}=1 / 4$ and $v_{2}=3 / 4$. By assumption, $\mathcal{B}=\mathbb{R}^{+}$. Note that $I_{1}=[2 / 3,5 / 6]$ and $I_{2}=[13 / 6,44 / 18]$ are 123-gaps.

Let $k \in\{4,5\}$. If $I_{2} \subseteq B_{k}$ then there exists an $i \geq 3$ such that $(6 i-1) x_{k}<13 / 6$ and $(6 i+1) x_{k}>44 / 18$. As these are contradictory inequalities, we have

$$
I_{2} \not \subset B_{4} \text { and } I_{2} \nsubseteq B_{5} \text {. }
$$

Therefore, it follows from Lemma 12 that there exist integers $r, s$ such that $v_{4}=r / 4$ and $v_{5}=s / 4$.

We now make two observations about the integers $r$ and $s$. First, consider the time $t=2 / 3$. The positions of runners 4 and 5 at this time are $r / 6$ and $s / 6$, respectively. Since $t=2 / 3$ must be covered by either runner 4 or runner 5 , we must have either $6 \mid r$ or $6 \mid s$. For our second observation, we consider $I_{2}$. Since $I_{2}$ is a 123-gap of length 5/18, we can conclude, by Lemma 4 , that $r \in\{5,6,7,8,9\}$. We consider each of these possible values of $r$ separately.

If $r=6$ then $I_{2}$ is a 1234-gap and we have a contradiction by (35). For each of the remaining cases we may assume $6 \mid s$. If $r=5$ then $[13 / 6,68 / 30]$ is a 1234-gap of length $1 / 10$, it follows that $s \in\{6,12\}$, and in either case we have a contradiction. If $r=7$ or $r=8$, then $I_{1}$ is a 1234-gap of length $1 / 6$, and runner 5 cannot possibly cover this gap as in either of these cases $s \geq 12$. If $r=9$ then [2/3,44/54] is a 1234-gap of length $x_{4} / 3$. 
In most of the remainder of this section we focus on times of the form $t=l+1 / 2$ where $l$ is a positive integer. These are times when runner 3 is at $1 / 2$, opposite the starting point. Note that if we know the location of runner 1 at such a time $t$ then we know the location of runner 2 at that time via $v_{1} t+v_{2} t=v_{3} t=t$. In particular, if runner 1 is in the interval $[1 / 6,1 / 3]$ at such a time $t$, then so is runner 2 , and the interval $[t, t+1 / 3]$ is a 0123-gap (note that we must use $v_{1}<1 / 6$ to ensure that $B_{0}$ does not intersect this 123-gap). The following lemma strengthens this observation.

Lemma 14. Assume $v_{1}+v_{2}=1$. Let $0 \leq \rho \leq 1 / 6$. If there exists a time $t$ satisfying $\left\{v_{3} t\right\}=1 / 2$ and

$$
\left\{v_{1} t\right\} \in\left[1 / 6+\rho v_{1}, 1 / 3-\rho v_{2}\right]
$$

then there exists a 0123-gap of length at least $1 / 3+\rho$.

Proof. Note that for $t$ satisfying the conditions of the lemma, $\left\{v_{2} t\right\}=1 / 2-\left\{v_{1} t\right\}$. Thus, the 123-gap containing $t$ starts either at the last time before time $t$ when runner 2 leaves $(-1 / 6,1 / 6)$ or the last time before $t$ when runner 1 leaves $(-1 / 6,1 / 6)$. These times are given by

$$
t-\left(\left\{v_{2} t\right\}-1 / 6\right) x_{2}=t-\left(1 / 3-\left\{v_{1} t\right\}\right) x_{2}
$$

and

$$
t-\left(\left\{v_{1} t\right\}-1 / 6\right) x_{1}
$$

respectively. Since the 123-gap in question ends at $t+1 / 3$, it has length at least $1 / 3+\rho$ if

$$
\min \left\{\left(\left\{v_{1} t\right\}-1 / 6\right) x_{1},\left(1 / 3-\left\{v_{1} t\right\}\right) x_{2}\right\} \geq \rho,
$$

which is equivalent to the condition in the lemma. Once again, it follows from the condition $v_{1}<1 / 6$ on the existence of runner 0 that this 123-gap is also a 0123-gap.

Corollary 15. Assume $v_{1}=p / q, v_{2}=(q-p) / q$ and $\mathcal{B}^{\prime}=\mathbb{R}^{+}$. There exists a 0123-gap of length at least $1 / 3$.

Proof. We apply Lemma 14 with $\rho=0$. It suffices to find a time of the form $t=l+1 / 2$ such that $\left\{v_{1} t\right\} \in[1 / 6,1 / 3]$. However, the set of positions of runner 1 at the times $t=l+1 / 2$ is simply a set of $q$ equidistributed points on the circle. Thus, if $q \geq 6$ one of them will lie in $[1 / 6,1 / 3]$. By Lemma 13 and the fact that $v_{1}<1 / 3$, the only remaining possibility is $v_{1}=1 / 5$, for which we find $v_{1}(1+1 / 2)=3 / 10 \in[1 / 6,1 / 3]$.

Corollary 16. If $v_{1}=p / q, v_{2}=(q-p) / q$ and $\mathcal{B}^{\prime}=\mathbb{R}^{+}$then $v_{4}<2$.

Proof. This follows from Corollary 15 and Lemma 4.

Lemma 17. Assume $v_{1}=p / q, v_{2}=(q-p) / q$ and $\mathcal{B}^{\prime}=\mathbb{R}^{+}$. There exist $r, s \in \mathbb{Z}$ such that $v_{4}=r / q$ and $v_{5}=s / q$.

Proof. By Corollary 15, there exists a 0123-gap that is neither covered entirely by runner 4 nor covered entirely by runner 5 . The result then follows directly from Lemma 12. 
In the very technical case analysis below, we will find it useful to have a better upper bound on $v_{4}$ than the one provided by Corollary 16. In the following lemma and table we get such an upper bound on $v_{4}$ by using the full power of Lemma 14 (rather than just $\rho=0)$.

Lemma 18. Assume $v_{1}=p / q, v_{2}=(q-p) / q$ and $\mathcal{B}^{\prime}=\mathbb{R}^{+}$. If $q \geq 12$ then $v_{4} \leq 3 / 2$. For each possible $(p, q)$ with $q \leq 17,\left\{v_{4}\right\}$ is bounded above by the number given in Table 1 .

Proof. In order to prove that $v_{4} \leq 3 / 2$ it suffices, by Lemma 4 , to show that there exists a 0123-gap $I$ such that $|I| \geq 4 / 9$. The set of positions of runner 1 at times $t=l+1 / 2$ is a set of $q$ points that is equidistributed in $[0,1)$. Thus, if we have

$$
1 / 6 \geq \rho v_{1}+\rho v_{2}+\frac{1}{q}=\rho+\frac{1}{q}
$$

then there exists such a position in the interval $\left[1 / 6+\rho v_{1}, 1 / 3-\rho v_{2}\right]$. Therefore, by Lemma 14 there exists a 0123-gap I satisfying

$$
|I| \geq 1 / 3+1 / 6-1 / q=\frac{q-2}{2 q}
$$

This is at least $4 / 9$ for $q \geq 18$.

For $q \leq 17$, we first note that the actual set of positions of runner 1 at times $l+1 / 2$ is $\{i / q: i=0, \ldots, q-1\}$ for $p$ even and $\{(2 i+1) / 2 q: i=0, \ldots, q-1\}$ for $p$ odd. Then for each possible pair $(p, q)$ we identify the position of runner 1 in $(1 / 6,1 / 3)$ for which the 0123-gap given by Lemma 14 is longest. This 0123-gap, in turn, gives an upper bound on $v_{4}$, by Lemma 4 . This upper bound is further reduced by noting that the denominator of $v_{4}$ is $q$. These calculations are organized in Table 1 . An inspection of the table shows that, in fact, $v_{4} \leq 3 / 2$ holds already for $q \geq 12$.

We now come to Part 3 of the proof of Proposition 11, the application of the main lemma. Once again, we consider times of the form $t=l+1 / 2$. As noted above, if runner 1 is in $[1 / 6,1 / 3]$ at such a time then $[t, t+1 / 3]$ is contained in a 0123-gap. If, in addition, runner 4 is in the interval $[1 / 6,1 / 2]$ at time $t$, then $\left[t, t+x_{4} / 3\right]$ is contained in a 01234-gap. Of course, runner 5 cannot cover this gap. Thus, we may conclude that there does not exist a time $t$ of the form $t=l+1 / 2$ such that $l$ is a positive integer and we have

$$
\begin{gathered}
\left\{v_{1} t\right\} \in[1 / 6,1 / 3], \text { and } \\
\left\{v_{4} t\right\} \in[1 / 6,1 / 2] .
\end{gathered}
$$

In other words, the set

$$
S=\left\{\left(\left\{v_{4}(l+1 / 2)\right\},\left\{v_{1}(l+1 / 2)\right\}\right): l=0,1, \ldots\right\}
$$

in the torus $T$ does not intersect the $1 / 3 \times 1 / 6$ rectangle determined by (36) and (37). 


\begin{tabular}{|c|c|c|c|c|c|c|}
\hline$q$ & $p$ & $\left\{v_{1} t\right\}$ & $x_{1}\left(\left\{v_{1} t\right\}-\frac{1}{6}\right)$ & $x_{2}\left(\frac{1}{3}-\left\{v_{1} t\right\}\right)$ & $|I|$ & $\left\{v_{4}\right\} \leq$ \\
\hline 5 & 1 & $\frac{3}{10}$ & $\frac{2}{3}$ & $\frac{1}{24}$ & $\frac{9}{24}$ & $\frac{3}{5}$ \\
\hline 6 & 1 & $\frac{3}{12}$ & $\frac{1}{2}$ & $\frac{1}{10}$ & $\frac{13}{30}$ & $\frac{3}{6}$ \\
\hline 7 & 1 & $\frac{3}{14}$ & $\frac{1}{3}$ & $\frac{5}{36}$ & $\frac{17}{36}$ & $\frac{2}{7}$ \\
\hline 7 & 2 & $\frac{2}{7}$ & $\frac{5}{12}$ & $\frac{1}{15}$ & $\frac{2}{5}$ & $\frac{4}{7}$ \\
\hline 8 & 1 & $\frac{3}{16}$ & $\frac{1}{6}$ & $\frac{1}{6}$ & $\frac{1}{2}$ & $\frac{2}{8}$ \\
\hline 9 & 1 & $\frac{5}{18}$ & 1 & $\frac{1}{16}$ & $\frac{19}{48}$ & $\frac{6}{9}$ \\
\hline 9 & 2 & $\frac{2}{9}$ & $\frac{1}{4}$ & $\frac{1}{7}$ & $\frac{10}{21}$ & $\frac{3}{9}$ \\
\hline 10 & 1 & $\frac{5}{20}$ & $\frac{5}{6}$ & $\frac{5}{54}$ & $\frac{23}{54}$ & $\frac{5}{10}$ \\
\hline 10 & 3 & $\frac{5}{20}$ & $\frac{5}{18}$ & $\frac{5}{42}$ & $\frac{19}{42}$ & $\frac{4}{10}$ \\
\hline 11 & 1 & $\frac{5}{22}$ & $\frac{2}{3}$ & $\frac{7}{60}$ & $\frac{27}{60}$ & $\frac{5}{11}$ \\
\hline 11 & 2 & $\frac{2}{11}$ & $\frac{1}{12}$ & $\frac{5}{27}$ & $\frac{5}{12}$ & $\frac{6}{11}$ \\
\hline 11 & 3 & $\frac{5}{22}$ & $\frac{2}{9}$ & $\frac{7}{48}$ & $\frac{23}{48}$ & $\frac{4}{11}$ \\
\hline 12 & 1 & $\frac{5}{24}$ & $\frac{1}{2}$ & $\frac{3}{22}$ & $\frac{31}{66}$ & $\frac{5}{12}$ \\
\hline 13 & 1 & $\frac{5}{26}$ & $\frac{1}{3}$ & $\frac{11}{72}$ & $\frac{35}{72}$ & $\frac{4}{13}$ \\
\hline 13 & 2 & $\frac{3}{13}$ & $\frac{5}{12}$ & $\frac{4}{33}$ & $\frac{15}{33}$ & $\frac{6}{13}$ \\
\hline 13 & 3 & $\frac{5}{26}$ & $\frac{1}{9}$ & $\frac{11}{60}$ & $\frac{4}{9}$ & $\frac{6}{13}$ \\
\hline 13 & 4 & $\frac{3}{13}$ & $\frac{5}{24}$ & $\frac{4}{27}$ & $\frac{13}{27}$ & $\frac{4}{13}$ \\
\hline 14 & 1 & $\frac{5}{28}$ & $\frac{1}{6}$ & $\frac{1}{6}$ & $\frac{1}{2}$ & $\frac{4}{14}$ \\
\hline 14 & 3 & $\frac{7}{28}$ & $\frac{7}{18}$ & $\frac{7}{66}$ & $\frac{29}{66}$ & $\frac{7}{14}$ \\
\hline 15 & 1 & $\frac{7}{30}$ & 1 & $\frac{3}{28}$ & $\frac{37}{84}$ & $\frac{7}{15}$ \\
\hline 15 & 2 & $\frac{3}{15}$ & $\frac{1}{4}$ & $\frac{2}{13}$ & $\frac{19}{39}$ & $\frac{5}{15}$ \\
\hline 15 & 4 & $\frac{3}{15}$ & $\frac{1}{8}$ & $\frac{2}{11}$ & $\frac{11}{24}$ & $\frac{6}{15}$ \\
\hline 16 & 1 & $\frac{7}{32}$ & $\frac{5}{6}$ & $\frac{11}{90}$ & $\frac{41}{90}$ & $\frac{7}{16}$ \\
\hline 16 & 3 & $\frac{7}{32}$ & $\frac{5}{18}$ & $\frac{1}{78}$ & $\frac{37}{78}$ & $\frac{6}{16}$ \\
\hline 16 & 5 & $\frac{7}{32}$ & $\frac{1}{6}$ & $\frac{1}{2}$ & $\frac{5}{16}$ \\
\hline 17 & 1 & $\frac{7}{34}$ & $\frac{2}{3}$ & $\frac{1}{96}$ & $\frac{7}{17}$ \\
\hline 17 & 2 & $\frac{4}{17}$ & $\frac{7}{12}$ & $\frac{1}{84}$ & $\frac{4}{9}$ & $\frac{8}{17}$ \\
\hline 17 & 3 & $\frac{7}{34}$ & $\frac{2}{9}$ & $\frac{5}{72}$ & $\frac{6}{17}$ \\
\hline 17 & 4 & $\frac{4}{17}$ & $\frac{7}{24}$ & $\frac{1}{39}$ & $\frac{7}{17}$ \\
\hline 17 & 5 & $\frac{7}{34}$ & $\frac{2}{15}$ & $\frac{7}{15}$ & $\frac{7}{17}$ \\
\hline
\end{tabular}

Table 1: Upper bounds on $\left\{v_{4}\right\}$ for small values of $p$ and $q$ that follow from the argument given in Lemma 18. 
In fact, we are able to conclude that $S$ does not intersect a certain pentagon that is obtained by relaxing the conditions given in (36) and (37). We shall see that if $1 / 6 \leq$ $v_{1}<1 / 3$ and $(x, y) \in S$ then we cannot simultaneously have

$$
\begin{gathered}
x \geq \frac{1}{6}-\frac{v_{4}}{3}\left(1-x_{5}\right), \\
y \geq \frac{1}{6}-\frac{v_{1}}{3}\left(1-x_{5}\right), \\
y \leq \frac{1}{3}+\frac{v_{2}}{3}\left(1-x_{5}\right), \text { and } \\
x \leq \frac{5}{6}-v_{4}\left[\frac{x_{5}}{3}-\min \left\{\frac{1}{v_{1}}\left(y-\frac{1}{6}\right), \frac{1}{v_{2}}\left(\frac{1}{3}-y\right)\right\}\right]
\end{gathered}
$$

Furthermore, if $v_{1}<1 / 6$ and $(x, y) \in S$ then we can not have (39), (40), (41), (42) and

$$
y \leq \frac{5}{12}-\frac{v_{1}}{3}
$$

This will follow from observing that if $(x, y)$ satisfies these conditions then there exists a 01234-gap of length at least $x_{5} / 3$ near the time $t=l+1 / 2$ that determines $(x, y)$. To see that this is the case, first observe that conditions (39), (40) and (41) allow runners 4,1 , and 2 , respectively, to be behind $\frac{1}{6}$ at time $t$, but they guarantee that they will arrive at $1 / 6$ no later than time $t+\left(1-x_{5}\right) / 3$. The expression

$$
\min \left\{\frac{1}{v_{1}}\left(y-\frac{1}{6}\right), \frac{1}{v_{2}}\left(\frac{1}{3}-y\right)\right\}
$$

in (42) is positive for $1 / 6<y<1 / 3$ and measures for such $y$ the length of the portion of the 123-gap that precedes time $t$; it is negative for $y<1 / 6$ or $y>1 / 3$ and its absolute value measures for such $y$ the time that will elapse from time $t$ until the beginning of the coming 123-gap.

Now, at time $t$ we are either in a 1234-gap, or, according to the conditions, such a gap will start as soon as runners 4, 1 and 2 have all passed 1/6. That 1234-gap will end when runner 3 or runner 4 arrive at $5 / 6$ - whichever comes first (it is easy to see that runners 1 and 2 cannot arrive at $5 / 6$ before runner 3 ). Conditions (39) thru (42) are designed so that the length of that 1234 -gap will be at least $x_{5} / 3$. Indeed, if the gap ends when runner 3 arrives at $5 / 6$, i.e. at time $t+1 / 3$, then conditions (39), (40) and (41) imply that its length is at least $x_{5} / 3$. If, on the other hand, the gap ends when runner 4 arrives at $5 / 6$ then the argument depends on the identity of the runner who was at $1 / 6$ when the gap started. It is easy to see that it could not be runner 3 . If it was runner 1 or runner 2 , then condition (42) implies that the length of the gap is at least $x_{5} / 3$. If it was runner 4 , then the length of the gap is $2 x_{4} / 3>x_{5} / 3$. In any case, the 1234-gap is too long for runner 5 to cover.

Condition (43) insures that, when $v_{1}<1 / 6$ runner 1 does not arrive at $5 / 12$ (i.e. runner 0 does not arrive at 5/6) before the end of the 1234-gap. Thus, when (43) is 
satisfied, we in fact have a 01234-gap that runner 5 cannot cover. So, it follows from our initial assumption $\mathcal{B}^{\prime}=\mathbb{R}^{+}$that $S$ does not intersect the pentagon defined by (39)-(43) for $v_{1}<1 / 6$ and $S$ does not intersect the pentagon defined by (39)-(42) for $1 / 6 \leq v_{1}<1 / 3$. We will refer to these pentagons as the expansion of the original $1 / 3 \times 1 / 6$ rectangle.

We proceed now to rewrite the above conditions in a manner that is convenient for an application of the main lemma. As $1<v_{4}<2$ (by Corollary 16) and $0<v_{1}<1$, we have

$$
\begin{gathered}
v_{4} t=v_{4}(l+1 / 2)=l v_{4}+\left\{v_{4}\right\} / 2+1 / 2 \\
v_{1} t=v_{1}(l+1 / 2)=l v_{1}+\left\{v_{1}\right\} / 2 .
\end{gathered}
$$

Let $G$ be the subgroup of $T$ generated by $\left(\left\{v_{4}\right\},\left\{v_{1}\right\}\right)$, and let $C$ be the coset

$$
C=G+\left(\left\{v_{4}\right\} / 2,\left\{v_{1}\right\} / 2\right) .
$$

Since $S=C+(1 / 2,0)$, our conditions can be rewritten in the form: $C$ does not intersect a translate (by $1 / 2$ in the $x$-coordinate) of the pentagon from the above discussion. More explicitly, let $P$ be the region on the torus defined by

$$
\begin{gathered}
\frac{2}{3}-\frac{v_{4}}{3}\left(1-x_{5}\right) \leq x \leq \frac{4}{3}-v_{4}\left[\frac{x_{5}}{3}-\min \left\{\frac{1}{v_{1}}\left(y-\frac{1}{6}\right), \frac{1}{v_{2}}\left(\frac{1}{3}-y\right)\right\}\right] \\
\frac{1}{6}-\frac{v_{1}}{3}\left(1-x_{5}\right) \leq y \leq \frac{1}{3}+\frac{v_{2}}{3}\left(1-x_{5}\right) .
\end{gathered}
$$

(note that we refer to points to the right of our $1 / 3 \times 1 / 6$ rectangle as having $x$-coordinate greater than 1). Let $P^{\prime}$ be the region defined by (46), (47), and (43). To reiterate, it follows from our initial assumption that $C \cap P=\emptyset$ for $1 / 6 \leq v_{1}<1 / 3$ and $C \cap P^{\prime}=\emptyset$ for $v_{1}<1 / 6$.

The pentagons $P$ and $P^{\prime}$ contain the rectangle $R:=[2 / 3,1] \times[1 / 6,1 / 3]$; the latter is the translate of the original rectangle that we had before the expansion. Figure 3 shows the shape of $P$ and its position relative to $R$; the shape of $P^{\prime}$ is similar, except the part above the line $y=5 / 12-v_{1} / 3$, if any, is cut off. We note for later reference that the sides of $P$ which are not parallel to the coordinate axes meet the respective horizontal sides of $P$ at points having first coordinate $x=4 / 3-v_{4} / 3$.

In fact, $P$ and $P^{\prime}$ contain a rectangle that is somewhat larger than $R$, namely

$$
R^{\prime}=\left[\frac{2}{3}-\frac{v_{4}}{3}\left(1-x_{5}\right), 1\right] \times\left[\frac{1}{6}, \frac{1}{3}\right] .
$$

The width of $R^{\prime}$ is $\alpha=1 / 3+v_{4}\left(1-x_{5}\right) / 3>v_{4} / 3$ and the height of $R$ is $\beta=1 / 6$. We now apply the main lemma to the group generated by $\left(\left\{v_{4}\right\},\left\{v_{1}\right\}\right)$ using these values of $\alpha$ and $\beta$. Clearly, if $G$ intersects every $\alpha \times \beta$ rectangle in $T$ then so does every coset of $G$; in particular, $C \cap R^{\prime} \neq \emptyset$, which is a contradiction. By the corollary to the main lemma, this implies the existence of a minimal element $(i / n, j / n) \in G \backslash\{(0,0)\}$ satisfying

$$
0 \leq i<\frac{1}{\beta}=6 \text { and }|j|<\frac{1}{\alpha}(1+\beta i)<\frac{3}{v_{4}}\left(1+\frac{i}{6}\right) \text {. }
$$




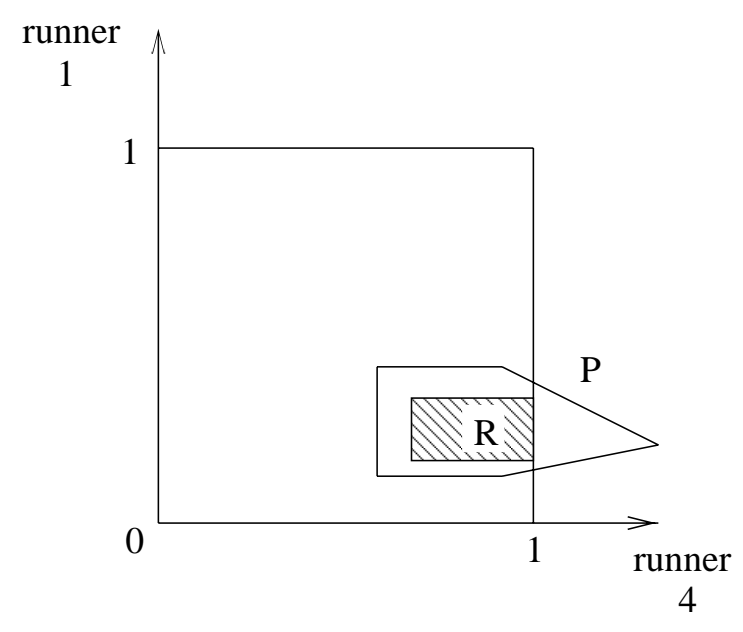

Figure 3: The pentagon $P$ and the rectangle $R$.

Thus, the values of $(i, j)$ that we must consider are

$$
i=0,1, \ldots, 5, \quad j=0, \pm 1, \ldots, \pm\left(2+\left\lceil\frac{i}{2}\right\rceil\right) .
$$

Moreover, it follows from (48) that whenever we consider a pair $(i, j)$ with $j \neq 0$ we may assume

$$
v_{4}<\frac{6+i}{2|j|}
$$

Below we shall consider systematically every pair $(i, j)$ given in (49) and analyze the case it represents until a contradiction is reached. The starting point of such an analysis, which is dubbed Part 4 of the proof of Proposition 11, is the following information that we have concerning the location of the elements of the subgroup $G$ (see the discussion in Sections 2 and 3). To every $(i, j)$ there corresponds a collection $\mathcal{L}_{i, j}$ of equally spaced parallel line segments in the $[0,1)^{2}$ square (for the purpose of the following description of $\mathcal{L}_{i, j}$ we are thinking of $[0,1)^{2}$ as a square rather than as a torus). The common slope of these line segments is $j / i$. For $j \geq 0$, one of the line segments starts at $(0,0)$ corner of the square, and for $j<0$ one of the line segments starts at the $(0,1)$ corner of the square. There are $|j|$ line segments intersecting any horizontal line $y=y_{0}$ and there are $i$ line segments intersecting any vertical line $x=x_{0}$. The elements of $G$ appear periodically on the circles in the torus formed by the line segments in $\mathcal{L}_{i, j}$. The period is $i / q$ when measured horizontally, or equivalently $|j| / q$ when measured vertically. Note that $|G|=q$ because $G$ is generated by $\left(\left\{v_{4}\right\},\left\{v_{1}\right\}\right)$ and we have $v_{1}=p / q,(p, q)=1$, and, by Lemma $17, v_{4}=r / q$.

The generator $\left(\left\{v_{4}\right\},\left\{v_{1}\right\}\right)$ of $G$ must itself lie on one of the line segments in $\mathcal{L}_{i, j}$. The identity of the line segment which contains $\left(\left\{v_{4}\right\},\left\{v_{1}\right\}\right)$ determines, in a manner that we proceed to explain, the location of the coset $C=G+\left(\left\{v_{4}\right\} / 2,\left\{v_{1}\right\} / 2\right)$. Suppose first that $j \geq 0$. If $\left(\left\{v_{4}\right\},\left\{v_{1}\right\}\right)$ lies on the line segment starting at $(0,0)$, or any line segment in $\mathcal{L}_{i, j}$ that is removed an even number of lines from it, then $\left(\left\{v_{4}\right\} / 2,\left\{v_{1}\right\} / 2\right)$ also lies 
on a line segment in $\mathcal{L}_{i, j}$. It follows that in this case the coset $C$ also lies periodically on the circles formed by $\mathcal{L}_{i, j}$, with the same period as $G$. On the other hand, if the line segment containing $\left(\left\{v_{4}\right\},\left\{v_{1}\right\}\right)$ is removed an odd number of lines from the one starting at $(0,0)$, then $\left(\left\{v_{4}\right\} / 2,\left\{v_{1}\right\} / 2\right)$ lies halfway between two adjacent segments in $\mathcal{L}_{i, j}$. It follows that in this case the $\operatorname{coset} C$ lies on a system of line segments $\mathcal{L}_{i, j}^{\prime}$, obtained from $\mathcal{L}_{i, j}$ by a parallel shift that places the line segments in $\mathcal{L}_{i, j}^{\prime}$ halfway between those of $\mathcal{L}_{i, j}$. Again, the elements of $C$ appear periodically on the circles formed by $\mathcal{L}_{i, j}^{\prime}$ with the same period as $G$. We refer below to this case as the shifted case, and to the former case as the unshifted one. The same dichotomy appears when $j<0$. If $\left(\left\{v_{4}\right\},\left\{v_{1}\right\}\right)$ lies on the line segment closest to $(0,0)$, or any line segment that is removed an even number of lines from it, we are in the shifted case; otherwise, we are in the unshifted case.

For every possible pair $(i, j)$, we try to determine on which of the line segments in $\mathcal{L}_{i, j}$ the generator $\left(\left\{v_{4}\right\},\left\{v_{1}\right\}\right)$ may lie. Such a determination, using tools that we will describe shortly, will enable us to conclude that for certain $(i, j)$ pairs only the shifted or only the unshifted case is possible. In some cases, neither is possible, leading to an immediate contradiction. In some other cases, however, we will have to consider both the shifted and the unshifted variant. In any case, placing $\left(\left\{v_{4}\right\},\left\{v_{1}\right\}\right)$ on a certain line segment yields a linear equation that $\left\{v_{4}\right\},\left\{v_{1}\right\}$ must satisfy, which allows us to express $v_{4}$ in terms of $v_{1}$.

The tools that we use to determine where $\left(\left\{v_{4}\right\},\left\{v_{1}\right\}\right)$ may lie are the bounds on its coordinate values given by $v_{4}>1$, Lemma 18, Table 1 and (50) for the first coordinate and by $0<v_{1}<1 / 3$ for the second. An additional tool is available for pairs $(i, j)$ where $i$ and $j$ are not relatively prime. In this case the line segments in $\mathcal{L}_{i, j}$ form more than one circle on the torus, and the generator of $G$ must not lie on the circle through the origin, for if it did the whole subgroup $G$ would lie there. Using these tools, we classify the pairs $(i, j)$ into four classes: $\mathrm{S}$ (shifted), $\mathrm{U}$ (unshifted), $\mathrm{N}$ (neither) and $\mathrm{SU}$ (shifted or unshifted). We present the classification in Table 2, together with an indication of the number of the case below where each pair $(i, j)$, or shifted/unshifted variant of it, is treated.

Since the classification in Table 2 is obtained by a straightforward and repetitive application of the tools described above, we present the details of the argument for only a few examples.

$(i, j)=(1,-1)$ : In this case $\mathcal{L}_{i, j}$ consists of only one line segment given by $x+y=1$. Thus, we must have $\left\{v_{4}\right\}+\left\{v_{1}\right\}=1$. Since $v_{1}<1 / 3$, we must have $v_{4}>5 / 3$. By Lemma 18 and an inspection of Table 1 this is never the case.

$(i, j)=(1,3)$ : In this case $\mathcal{L}_{i, j}$ consists of three line segments of slope 3 starting at $(0,0),(1 / 3,0)$ and $(2 / 3,0)$, respectively. By $(50)$ we have $v_{4}<7 / 6$, which implies that $\left(\left\{v_{4}\right\},\left\{v_{1}\right\}\right)$ can only lie on the first of these line segments. Thus we are in the unshifted case.

$(i, j)=(2,2)$ : Here $\mathcal{L}_{i, j}$ is the collection of line segments starting at $(0,0),(0,1 / 2)$ and $(1 / 2,0)$ having slope 1 . Furthermore, $2 \mid q$. Since 2 and 2 are not relatively prime, the generator of $G$ cannot lie on the segment through $(0,0)$. Since $v_{1}<1 / 3$ the generator of $G$ cannot lie on the second line in $\mathcal{L}_{i, j}$. The only remaining possibility is for it to lie on 


\begin{tabular}{|c|c|c|c|c|c|c|}
\hline$j \backslash^{i}$ & 0 & 1 & 2 & 3 & 4 & 5 \\
\hline 5 & & & & & & $\mathrm{~S}_{2}$ \\
\hline 4 & & & & $\mathrm{U}_{3}$ & $\mathrm{~S}_{2}$ & $\mathrm{~S}_{7} \mathrm{U}_{3}$ \\
\hline 3 & & $\mathrm{U}_{3}$ & $\mathrm{U}_{3}$ & $\mathrm{~S}_{2}$ & $\mathrm{~S}_{3} \mathrm{U}_{7}$ & $\mathrm{~S}_{5} \mathrm{U}_{5}$ \\
\hline 2 & $\mathrm{~N}$ & $\mathrm{~S}_{3} \mathrm{U}_{11}$ & $\mathrm{~N}$ & $\mathrm{U}_{3}$ & $\mathrm{~S}_{6}$ & $\mathrm{~S}_{4} \mathrm{U}_{6}$ \\
\hline 1 & $\mathrm{~N}$ & $\mathrm{U}_{12}$ & $\mathrm{U}_{10}$ & $\mathrm{U}_{4}$ & $\mathrm{~S}_{8} \mathrm{U}_{4}$ & $\mathrm{~S}_{4} \mathrm{U}_{7}$ \\
\hline 0 & & $\mathrm{~N}$ & $\mathrm{~N}$ & $\mathrm{~N}$ & $\mathrm{~S}_{1}$ & $\mathrm{~S}_{1}$ \\
\hline-1 & $\mathrm{~N}$ & $\mathrm{~N}$ & $\mathrm{~S}_{7}$ & $\mathrm{~S}_{4}$ & $\mathrm{~S}_{6}$ & $\mathrm{~S}_{7} \mathrm{U}_{7}$ \\
\hline-2 & $\mathrm{~N}$ & $\mathrm{~S}_{9}$ & $\mathrm{~S}_{7}$ & $\mathrm{~S}_{3}$ & $\mathrm{~S}_{6}$ & $\mathrm{~S}_{6} \mathrm{U}_{4}$ \\
\hline-3 & & $\mathrm{~N}$ & $\mathrm{~S}_{7}$ & $\mathrm{~S}_{2} \mathrm{U}_{2}$ & $\mathrm{~S}_{3} \mathrm{U}_{7}$ & $\mathrm{~S}_{5} \mathrm{U}_{5}$ \\
\hline-4 & & & & $\mathrm{~S}_{3}$ & $\mathrm{~S}_{2}$ & $\mathrm{~S}_{3} \mathrm{U}_{7}$ \\
\hline-5 & & & & & & $\mathrm{~S}_{2} \mathrm{U}_{2}$ \\
\hline
\end{tabular}

Table 2: The classification of pairs $(i, j)$ as shifted $(\mathrm{S})$, unshifted $(\mathrm{U})$, neither $(\mathrm{N})$ or either (SU). The subscript of an entry is the case in which the entry is treated.

the line $v_{4}=3 / 2+v_{1}$. It follows from Lemma 18 and an inspection of Table 1 that this is not the case.

$(i, j)=(3,3):$ In this case $\mathcal{L}_{i, j}$ consists of the line segments of slope one through $(0,2 / 3),(0,1 / 3),(0,0),(1 / 3,0)$ and $(2 / 3,0)$. Since $i$ and $j$ are not relatively prime, the generator of $G$ cannot lie on the line segment containing $(0,0)$. The restriction $v_{1}<1 / 3$ implies that it does not lie on the first two line segments listed above. The restriction $v_{4}<3 / 2$ that follows from (50) implies that it does not lie on the last of the line segments. Thus $\left(\left\{v_{4}\right\},\left\{v_{1}\right\}\right)$ can only lie on the line segment starting at $(1 / 3,0)$, and we are in the shifted case.

We now embark on Part 5 of the proof of Proposition 11, in which we treat all pairs $(i, j)$ that appear in Table 2 , with the exception of the pair $(1,1)$. Note that some of the pairs from (49) were actually handled in the pre-processing above (i.e. Part 4 of the proof of Proposition 11). The pairs are grouped into cases.

Case 5.2.1. $(i, 0), i=4,5$

In these cases the generator $\left(\left\{v_{4}\right\},\left\{v_{1}\right\}\right)$ of $G$ must lie on the line $y=1 / i$, which implies that $p / q=1 / i$ and hence $q=i$, contradicting (14).

Case 5.2.2. $(i, \pm i), i=3,4,5$

In all of these cases, both $\mathcal{L}_{i, j}$ and $\mathcal{L}_{i, j}^{\prime}$ contain a line segment whose intersection with $R$ has full vertical length of $1 / 6$. Thus, our assumption that $C \cap R=\emptyset$ implies that $q<6 i$. Since in these cases we must have $i \mid q$, we actually have $q \leq 5 i$. On the other hand (14) implies $q \geq i^{2}+2 i$. These two bounds are contradictory for $i=4,5$, and leave us with only $q=15$ for $i=3$.

In the case of the pair $(3,3)$, which can only be shifted, we have $\left\{v_{4}\right\}=1 / 3+v_{1}=$ $(5+p) / 15$. It follows from this equation and Table 1 that $p=1$. However, in this case $(1,1 / 6) \in C \cap R$. 


\begin{tabular}{|c|c|c|c|}
\hline$(1,2)_{S}$ & {$[4,11]$} & $\frac{q+p}{2 q}$ & $p, q$ odd \\
\hline$(3,2)_{U}$ & {$[11,11]$} & $\frac{3 p}{2 q}$ & $p$ even \\
\hline$(3,-2)_{S}$ & {$[11,11]$} & $\frac{q-3 p}{2 q}$ & $p, q$ odd \\
\hline$(1,3)_{U}$ & {$[6,17]$} & $\frac{p}{3 q}$ & $3 \mid p$ \\
\hline$(2,3)_{U}$ & {$[11,17]$} & $\frac{2 p}{3 q}$ & $3 \mid p$ \\
\hline$(4,3)_{S}$ & $\emptyset$ & & \\
\hline$(4,-3)_{S}$ & $\emptyset$ & & \\
\hline$(3,4)_{U}$ & {$[19,23]$} & $\frac{3 p}{4 q}$ & $4 \mid p$ \\
\hline$(3,-4)_{S}$ & {$[19,23]$} & $\frac{q-3 p}{4 q}$ & $p \equiv-q(\bmod 4)$ \\
\hline$(5,4)_{U}$ & $\emptyset$ & & \\
\hline$(5,-4)_{S}$ & $\emptyset$ & & \\
\hline
\end{tabular}

Table 3: Conditions on $p$ and $q$ for the pairs in Case 5.2.3.

In the case of $(3,-3)_{S}$, we have $\left\{v_{4}\right\}=1 / 3-v_{1}=(5-p) / 15$. For $p=1,2,4$ we find the points $(14 / 15,7 / 30),(9 / 10,4 / 15)$ and $(5 / 6,1 / 3)$, respectively, in $C \cap R$.

In the case of $(3,-3)_{U}$, we have $\left\{v_{4}\right\}=2 / 3-v_{1}=(10-p) / 15$. Table 1 indicates that this is possible only for $p=4$. But in this case $(4 / 5,1 / 5) \in C \cap R$.

Case 5.2.3. $(1,2)_{S},(3,2)_{U},(3,-2)_{S},(1,3)_{U},(2,3)_{U},(4,3)_{S},(4,-3)_{S},(3,4)_{U},(3,-4)_{S}$, $(5,4)_{U},(5,-4)_{S}$

In all of these cases either $\mathcal{L}_{i, j}$ or $\mathcal{L}_{i, j}^{\prime}$ contains a segment whose intersection with $R$ has full vertical length of $1 / 6$. Thus, our assumption that $C \cap R=\emptyset$ implies that $q<6|j|$ in each case. Together with the lower bound on $q$ given by (14), this leaves only a certain (possibly empty) interval of possible values of $q$ to be considered in each case. The second column in Table 3 specifies that interval. The identification of the line segment on which $\left(\left\{v_{4}\right\},\left\{v_{1}\right\}\right)$ lies gives, in each remaining case, an expression for $\left\{v_{4}\right\}$ in terms of $v_{1}$, and hence in terms of $p$ and $q$, which is listed in the third column. This expression and Lemma 17 imply certain restrictions on the possible values of $p$ and $q$, which are listed in the fourth column.

We now note that some of the remaining cases can be eliminated by other considerations. For $(1,2)_{S}$, it follows from Table 1 that the only cases in which $\left\{v_{4}\right\}=(q+p) / 2 q$ and $p, q$ odd is a possibility are $q=5, p=1$ and $q=9, p=1$. In the cases $(3, \pm 4)$, we have the upper bound $v_{4}<9 / 8$ from (50). In the case of $(3,4)_{U}$ this becomes $3 p / 4 q<1 / 8$ (i.e. $p<q / 6)$ which is incompatible with $4 \mid p$ and $q \leq 23$. In the case of $(3,-4)_{S}$ this bound is $(q-3 p) / 4 q<1 / 8$ (i.e. $p>q / 6$ ); in conjunction with the restrictions given in Table 3 for this case, only $q=19, p=5$ and $q=23, p=5$ remain possible.

What remains is treated in Table 4. For each pair and every choice of $p$ and $q$ consistent with the above restrictions, we specify an element of $C$ that is either in $R$ or, when no element of $C \cap R$ exists, in $P$. The instances in which an element of $P \backslash R$ is given satisfy $v_{1}>1 / 6$, and so the existence of an element of $C \cap P$ is a contradiction (to verify that 


\begin{tabular}{|c|c|c|c|c|c|c|c|}
\hline$q$ & $p$ & $(1,2)_{S}$ & $(3,2)_{U}$ & $(3,-2)_{S}$ & $(1,3)_{U}$ & $(2,3)_{U}$ & $(3,-4)_{S}$ \\
\hline 5 & 1 & $\left(\frac{9}{10}, \frac{3}{10}\right)$ & & & & & \\
\hline 9 & 1 & $\left(\frac{5}{6}, \frac{1}{6}\right)$ & & & & & \\
\hline 10 & 3 & & & & $\left(\frac{3}{4}, \frac{1}{4}\right)$ & & \\
\hline 11 & 1 & & & $\left(\frac{10}{11}, \frac{5}{22}\right)$ & & & \\
\hline 11 & 2 & & $\left(\frac{17}{22}, \frac{2}{11}\right)$ & & & & \\
\hline 11 & 3 & & & $\left(\frac{17}{22}, \frac{7}{22}\right)$ & $\left(\frac{17}{22}, \frac{7}{22}\right)$ & $\left(\frac{9}{11}, \frac{5}{22}\right)$ & \\
\hline 13 & 3 & & & & $\left(\frac{19}{26}, \frac{5}{26}\right)$ & $\left(\frac{11}{13}, \frac{7}{26}\right)$ & \\
\hline 14 & 3 & & & & $\left(\frac{3}{4}, \frac{1}{4}\right)$ & $\left(\frac{11}{14}, \frac{5}{28}\right)$ & \\
\hline 16 & 3 & & & & $\left(\frac{25}{32}, \frac{11}{32}\right)^{*}$ & $\left(\frac{13}{16}, \frac{7}{12}\right)$ & \\
\hline 17 & 3 & & & & $\left(\frac{25}{34}, \frac{7}{34}\right)$ & $\left(\frac{5}{17}, \frac{11}{34}\right)$ & \\
\hline 19 & 5 & & & & & & $\left(\frac{25}{38}, \frac{11}{38}\right)^{*}$ \\
\hline 23 & 5 & & & & & & $\left(\frac{21}{23}, \frac{13}{46}\right)$ \\
\hline
\end{tabular}

Table 4: Elements of $C$ that are in $R$ or $P$ for the remaining cases in Case 5.2.3.

\begin{tabular}{|c|c|c|c|c|c|}
\hline$(3,1)_{U}$ & $(3,-1)_{S}$ & $(4,1)_{U}$ & $(5,1)_{S}$ & $(5,2)_{S}$ & $(5,-2)_{U}$ \\
\hline$[6,8]$ & {$[6,8]$} & {$[8,11]$} & {$[10,14]$} & $\emptyset$ & $\emptyset$ \\
\hline$\frac{3 p}{q}$ & $\frac{q-3 p}{q}$ & $\frac{4 p}{q}$ & $\frac{5 p-q}{q}$ & & \\
\hline
\end{tabular}

Table 5: Conditions on $p$ and $q$ for the pairs in Case 5.2.4.

these elements lie in $P$ consider (46) and (47), taking into account the actual values of $v_{2}$ and $v_{4}$ and the lower bound $\left.1-x_{5}>1-x_{4}\right)$. These instances are marked with an asterisk.

Case 5.2.4. $(3,1)_{U},(3,-1)_{S},(4,1)_{U},(5,1)_{S},(5,2)_{S},(5,-2)_{U}$

In these cases either $\mathcal{L}_{i, j}$ or $\mathcal{L}_{i, j}^{\prime}$ (whichever is applicable) has a segment whose intersection with $R$ has full horizontal length of $1 / 3$. Thus, it follows from $C \cap R=\emptyset$ that $q<3 i$. Together with the lower bound on $q$ given by (14), this leaves only a certain (possibly empty) interval of possible values of $q$ to be considered in each case. The second row of Table 5 specifies that interval. The line in $\mathcal{L}_{i, j}$ on which $\left(\left\{v_{4}\right\},\left\{v_{1}\right\}\right)$ lies gives a relationship between $\left\{v_{4}\right\}$ and $v_{1}$. This yields an expression for $\left\{v_{4}\right\}$ in terms of $p$ and $q$ which is given in the third row of Table 5 .

Note that some of the remaining cases are eliminated by a consideration of the third row of Table 5 alone. In particular, in the case $(4,1)_{U}$ we must have $4 p<q$ and in the case $(5,1)_{S}$ we must have $q<5 p$. The remaining cases are treated in Table 6 . Some of these cases can be eliminated by noting that the bound on $\left\{v_{4}\right\}$ given in Table 1 is incompatible with the expression for $\left\{v_{4}\right\}$ given in the third row of Table 5 . These cases are marked with a -- in the table. For most of the other cases we are able to specify an element of $C \cap R$. In two of the cases we must resort to identifying an element of $C \cap P$. These elements are marked with an asterisk, and in these cases $v_{1}>1 / 6$, and therefore an element of $C \cap P$ is sufficient to give a contradiction. 


\begin{tabular}{|c|c|c|c|c|c|}
\hline$q$ & $p$ & $(3,1)_{U}$ & $(3,-1)_{S}$ & $(4,1)_{U}$ & $(5,1)_{S}$ \\
\hline 6 & 1 & $\left(\frac{3}{4}, \frac{1}{4}\right)$ & $\left(\frac{3}{4}, \frac{1}{4}\right)$ & & \\
\hline 7 & 1 & -- & -- & & \\
\hline 7 & 2 & -- & $\left(\frac{9}{14}, \frac{2}{7}\right)^{*}$ & & \\
\hline 8 & 1 & -- & -- & -- & \\
\hline 9 & 1 & & & $\left(\frac{2}{3}, \frac{1}{6}\right)$ & \\
\hline 9 & 2 & & & -- & \\
\hline 10 & 1 & & & $\left(1, \frac{1}{4}\right)$ & \\
\hline 10 & 3 & & & & -- \\
\hline 11 & 1 & & & $\left(\frac{10}{11}, \frac{5}{22}\right)$ & \\
\hline 11 & 2 & & & -- & \\
\hline 11 & 3 & & & & $\left(\frac{7}{11}, \frac{5}{22}\right)^{*}$ \\
\hline 13 & 3 & & & & $\left(\frac{11}{13}, \frac{7}{26}\right)$ \\
\hline 13 & 4 & & & & -- \\
\hline 14 & 3 & & & & $\left(\frac{3}{4}, \frac{1}{4}\right)$ \\
\hline
\end{tabular}

Table 6: Treating the remaining cases in Case 5.2.4.

\begin{tabular}{|c|c|c|c|c|}
\hline$(4,-1)_{S}$ & $(4,2)_{S}$ & $(4,-2)_{S}$ & $(5,2)_{U}$ & $(5,-2)_{S}$ \\
\hline$\frac{1}{6}$ & $\frac{1}{4}$ & $\frac{1}{4}$ & $\frac{1}{6}$ & $\frac{1}{6}$ \\
\hline$[8,23]$ & {$[14,15]$} & {$[14,15]$} & {$[17,29]$} & {$[17,29]$} \\
\hline$\frac{q-4 p}{q}$ & $\frac{4 p-q}{2 q}$ & $\frac{q-4 p}{2 q}$ & $\frac{5 p}{2 q}$ & $\frac{q-5 p}{2 q}$ \\
\hline$p<\frac{q}{4}$ & $q$ even & $q$ even & $p$ even & $p, q$ odd \\
$q \geq 12 \Rightarrow p \geq \frac{q}{8}$ & $p>\frac{q}{4}$ & $p<\frac{q}{4}$ & $p \leq \frac{q}{5}$ & $p<\frac{q}{5}$ \\
\hline
\end{tabular}

Table 7: Conditions on $p$ and $q$ for the pairs in Case 5.2.6.

Case 5.2.5. $(5, \pm 3)_{U},(5, \pm 3)_{S}$

For these pairs, both $\mathcal{L}_{i, j}$ and $\mathcal{L}_{i, j}^{\prime}$ have line segments whose intersection with $R$ has length 2/9 (measured horizontally). Thus $C \cap R=\emptyset$ implies $q<45 / 2$. On the other hand, (14) implies $q \geq 23$, a contradiction.

Case 5.2.6. $(4,-1)_{S},(4,2)_{S},(4,-2)_{S},(5,2)_{U},(5,-2)_{S}$

Table 7 indicates which values of $p$ and $q$ need to be considered for each of these pairs. The second row shows the length (measured horizontally) of the intersection of a suitable line segment in $\mathcal{L}_{i, j}$ or $\mathcal{L}_{i, j}^{\prime}$ (whichever is applicable) with $R$. The third row specifies the interval to which $q$ must belong, based on the above intersection length and (14). The fourth row gives the expression for $\left\{v_{4}\right\}$ in terms of $p$ and $q$. The fifth row contains conditions on $p$ and $q$ that are obtained from the expression for $\left\{v_{4}\right\}$ in the fourth row and Lemmas 17 and 18 .

In the case of $(4,2)_{S}$, the conditions obtained on $p$ and $q$ are contradictory. The remaining pairs are treated in tables on page 33. As in previous cases, a - - indicates 
incompatibility between the expression in the fourth row of Table 7 and the upper bounds on $\left\{v_{4}\right\}$ given in Table 1 . In all other cases (that satisfy the conditions given in the fifth row of Table 7) we present an element of $C \cap P$. An asterisk indicates that this is an element of $P \backslash R$ and $v_{1}>1 / 6$; a double asterisk indicates that this is an element of $P^{\prime} \backslash R$.

Case 5.2.7. $(2,-1)_{S},(5,1)_{U},(5,-1)_{S},(5,-1)_{U},(2,-2)_{S},(2,-3)_{S},(4,3)_{U},(4,-3)_{U}$, $(5,4)_{S},(5,-4)_{U}$

In these cases, either $\mathcal{L}_{i, j}$ or $\mathcal{L}_{i, j}^{\prime}$ (whichever is applicable) has a segment that intersects the $x=1$ line at some point $y$ between $1 / 6$ and $1 / 3$. We are going to take advantage of the continuation of that segment that still lies in $P$ (and $P^{\prime}$ ). Of course, the exact length of that continuation depends on $v_{1}, v_{2}, v_{4}$ and $x_{5}$. We shall be satisfied with a lower bound on the length of this extension, obtained by replacing the upper bound on $x$ given in (46) by the more restrictive upper bound (for $1 / 6 \leq y \leq 1 / 3$ )

$$
x \leq 1+\min \left\{3\left(y-\frac{1}{6}\right), \frac{1}{3}-y\right\} .
$$

To obtain (51) we used the estimates $v_{4} x_{5}<1, v_{4}>1, v_{1}<1 / 3$ and $v_{2}<1$.

The second column of Table 8 contains the right endpoint of the extension of line segment we are interested in (i.e. the largest $x$ of a point $(x, y)$ on the extension of the line segment that satisfies (51). The third column contains a lower bound on the (horizontal) length of the intersection of a circle occupied by $C$ with $P^{\prime}$. In all but one case, this lower bound is given by the union of the intersection of the given line segment with $R$ and its continuation to the right (as defined by (51)). In the case of $(5,-1)_{U}$ we use the intersection of the line segment with $R$, the extension to the right of $R$, and an extension to the left of $R$. In the $(5,-1)_{U}$ case, we observe that the point $(5 / 9,13 / 45)$ on the line $y=2 / 5-x / 5$ belongs to $P^{\prime}$. Indeed, the generator $\left(\left\{v_{4}\right\},\left\{v_{1}\right\}\right)$ lies on the same line, and since $v_{1}<1 / 3$ it follows that $v_{4}>4 / 3$ and hence $x_{5}<x_{4}<3 / 4$. This implies that $2 / 3-v_{4}\left(1-x_{5}\right) / 3<5 / 9$. Thus the line segment in question has an extension of length at least $1 / 9$ to the left of $R$. The fourth column indicates the (possibly empty) interval to which $q$ must belong, based on the above intersection length and (14). The fifth and sixth column, respectively, contain expressions for $\left\{v_{4}\right\}$ in terms of $p$ and $q$ and conditions on $p$ and $q$ obtained thereby.

The pairs that were not eliminated in the above table are treated in the tables on page 35 , using the same conventions as in previous cases.

Case 5.2.8. $(4,1)_{S}$

In this case the generator $\left(\left\{v_{4}\right\},\left\{v_{1}\right\}\right)$ is on the line $y=1 / 4+x / 4$. This has several implications. First, $v_{1}>1 / 4>1 / 6$, and so, by assumption, $C \cap P=\emptyset$. Second, $x_{1}<4$ implies that in order to cover the first 123-gap we must have

$$
x_{5}<\frac{x_{1}}{5}<\frac{4}{5} .
$$




\begin{tabular}{|c|c|c|c|c|c|c|c|c|}
\hline \multicolumn{9}{|c|}{$(4,-1)_{S}$} \\
\hline$q$ & $p$ & & $q$ & $p$ & & $q$ & $p$ & \\
\hline 8 & 1 & -- & 14 & 3 & $\left(\frac{11}{14}, \frac{5}{28}\right)$ & 21 & 4 & $\left(\frac{31}{42}, \frac{4}{21}\right)$ \\
\hline 9 & 1 & $\left(\frac{5}{6}, \frac{1}{6}\right)$ & 15 & 2 & -- & 21 & 5 & $\left(\frac{5}{6}, \frac{1}{6}\right)$ \\
\hline 9 & 2 & $\left(\frac{11}{18}, \frac{2}{9}\right)^{*}$ & 16 & 3 & $\left(\frac{5}{8}, \frac{7}{32}\right)^{*}$ & 22 & 3 & $\left(\frac{15}{22}, \frac{9}{44}\right)$ \\
\hline 10 & 1 & -- & 17 & 3 & $\left(\frac{23}{34}, \frac{7}{34}\right)$ & 22 & 5 & $\left(\frac{15}{22}, \frac{9}{44}\right)$ \\
\hline 11 & 1 & -- & 17 & 4 & $\left(\frac{27}{34}, \frac{3}{17}\right)$ & 23 & 3 & $\left(\frac{33}{46}, \frac{9}{46}\right)$ \\
\hline 11 & 2 & $\left(\frac{17}{22}, \frac{2}{11}\right)$ & 19 & 3 & $\left(\frac{29}{38}, \frac{7}{38}\right)$ & 23 & 4 & $\left(\frac{37}{46}, \frac{4}{23}\right)$ \\
\hline 13 & 2 & $\left(\frac{15}{26}, \frac{3}{13}\right)^{* *}$ & 19 & 4 & $\left(\frac{25}{38}, \frac{4}{19}\right)^{*}$ & 23 & 5 & $\left(\frac{33}{46}, \frac{9}{46}\right)$ \\
\hline 13 & 3 & $\left(\frac{19}{26}, \frac{5}{26}\right)$ & 20 & 3 & $\left(\frac{4}{5}, \frac{7}{40}\right)$ & & & \\
\hline
\end{tabular}

\begin{tabular}{|c|c|c|}
\hline \multicolumn{3}{|c|}{$(4,-2)_{S}$} \\
\hline$q$ & $p$ & \\
\hline 14 & 1 & -- \\
\hline 14 & 3 & $\left(\frac{3}{4}, \frac{1}{4}\right)$ \\
\hline
\end{tabular}

\begin{tabular}{|c|c|c|c|c|c|c|c|c|}
\hline \multicolumn{10}{|c|}{$(5,2)_{U}$} \\
\hline$q$ & $p$ & & $q$ & $p$ & & $q$ & $p$ & \\
\hline 17 & 2 & $\left(\frac{25}{34}, \frac{5}{17}\right)$ & 23 & 2 & $\left(\frac{35}{46}, \frac{7}{23}\right)$ & 27 & 2 & $\left(\frac{5}{6}, \frac{1}{3}\right)$ \\
\hline 19 & 2 & $\left(\frac{25}{38}, \frac{5}{19}\right)^{* *}$ & 23 & 4 & $\left(\frac{15}{23}, \frac{6}{23}\right)^{*}$ & 27 & 4 & $\left(\frac{20}{27}, \frac{8}{27}\right)$ \\
\hline 21 & 2 & $\left(\frac{5}{6}, \frac{1}{3}\right)$ & 25 & 2 & $\left(\frac{7}{10}, \frac{7}{25}\right)$ & 29 & 2 & $\left(\frac{45}{58}, \frac{9}{29}\right)$ \\
\hline 21 & 4 & $\left(\frac{5}{7}, \frac{2}{7}\right)$ & 25 & 4 & $\left(\frac{4}{5}, \frac{8}{25}\right)$ & 29 & 4 & $\left(\frac{20}{29}, \frac{8}{29}\right)$ \\
\hline
\end{tabular}

\begin{tabular}{|c|c|c|c|c|c|c|c|c|}
\hline \multicolumn{10}{|c|}{$(5,-2)_{S}$} \\
\hline$q$ & $p$ & & $q$ & $p$ & $q$ & $p$ & \\
\hline 17 & 1 & $\left(\frac{16}{17}, \frac{11}{34}\right)$ & 23 & 1 & $\left(\frac{35}{46}, \frac{9}{46}\right)^{*}$ & 27 & 5 & $\left(\frac{5}{6}, \frac{1}{6}\right)$ \\
\hline 17 & 3 & $\left(\frac{25}{34}, \frac{7}{34}\right)$ & 23 & 3 & $\left(\frac{15}{23}, \frac{11}{66}\right)^{* *}$ & 29 & 1 & $\left(\frac{20}{20}, \frac{13}{58}\right)$ \\
\hline 19 & 1 & $\left(\frac{25}{38}, \frac{9}{38}\right)^{* *}$ & 25 & 1 & $\left(\frac{4}{5}, \frac{9}{50}\right)$ & 29 & 3 & $\left(\frac{45}{58}, \frac{11}{58}\right)$ \\
\hline 19 & 3 & $\left(\frac{15}{19}, \frac{7}{38}\right)$ & 25 & 3 & $\left(\frac{7}{10}, \frac{11}{50}\right)$ & 29 & 5 & $\left(\frac{20}{29}, \frac{13}{58}\right)$ \\
\hline 21 & 1 & $\left(\frac{5}{7}, \frac{3}{14}\right)$ & 27 & 1 & $\left(\frac{5}{6}, \frac{1}{6}\right)$ & & & \\
\hline
\end{tabular}




\begin{tabular}{|c|c|c|c|c|c|}
\hline$(2,-1)_{S}$ & $\frac{11}{10}$ & $\frac{4}{15}$ & {$[4,7]$} & $\frac{\frac{q-2 p}{q}}{q}$ & \\
\hline$(5,1)_{U}$ & $\frac{10}{9}$ & $\frac{5}{18}$ & {$[10,17]$} & $\frac{5 p}{q}$ & $q \geq 12 \Rightarrow p \leq \frac{q}{10}$ \\
\hline$(5,-1)_{S}$ & $\frac{25}{24}$ & $\frac{5}{24}$ & {$[10,23]$} & $\frac{q-5 p}{q}$ & $q \geq 12 \Rightarrow p \geq \frac{q}{10}$ \\
\hline$\overline{(5,-1)_{U}}$ & $\frac{17}{16}$ & $\frac{73}{144}$ & $\emptyset$ & & \\
\hline$(2,-2)_{S}$ & $\frac{17}{16}$ & $\frac{7}{48}$ & {$[8,13]$} & $\frac{q-2 p}{2 q}$ & $q$ even \\
\hline$(2,-3)_{S}$ & $\frac{23}{22}$ & $\frac{10}{99}$ & {$[11,19]$} & $\frac{q-2 p}{3 q}$ & $3 \nmid q q \equiv-q(\bmod 3)$ \\
\hline$(4,3)_{U}$ & $\frac{22}{21}$ & $\frac{50}{63}$ & {$[19,25]$} & $\frac{4 p}{3 q}$ & $3 \mid p$ \\
\hline$(4,-3)_{U}$ & $\frac{14}{13}$ & $\frac{22}{117}$ & {$[19,21]$} & $\frac{2 q-4 p}{3 q}$ & $p \equiv-q(\bmod 3) \quad p \geq q / 8$ \\
\hline$(5,4)_{S}$ & $\frac{55}{54}$ & $\frac{5}{27}$ & $\emptyset$ & & \\
\hline$(5,-4)_{U}$ & $\frac{35}{34}$ & $\frac{10}{51}$ & $\emptyset$ & & \\
\hline
\end{tabular}

Table 8: Conditions on $p$ and $q$ for the pairs in Case 5.2.7.

Third, $v_{1}=1 / 4+\left\{v_{4}\right\} / 4$ implies

$$
v_{4}=4 v_{1} .
$$

We observe (using (52), (53), and the fact that the vertical line $x=4 / 3-v_{4} / 3$ passes through two vertices of $P$ ) that the portion of the line segment $y=1 / 8+x / 4$ corresponding to

$$
\frac{2}{3}-\frac{v_{4}}{3}\left(1-x_{5}\right) \leq x \leq \frac{4}{3}-\frac{v_{4}}{3}
$$

is contained in $P$. Since $C$ has period $4 / q$ on this line segment we must have (again using (52) and (53))

$$
\begin{aligned}
\frac{4}{q} & >\frac{4}{3}-\frac{v_{4}}{3}-\left[\frac{2}{3}-\frac{v_{4}}{3}\left(1-x_{5}\right)\right] \\
& =\frac{2}{3}-\frac{v_{4}}{3} x_{5} \\
& >\frac{2}{3}-\frac{v_{4}}{3} \frac{x_{1}}{5} \\
& =\frac{2}{5} .
\end{aligned}
$$

This implies that $q<10$. On the other hand (14) implies $q \geq 8$. However, for $q=8,9$ there is no $p$ such that $1 / 4<p / q<1 / 3$.

Case 5.2.9. $(1,-2)_{S}$

In this case the generator $\left(\left\{v_{4}\right\},\left\{v_{1}\right\}\right)$ is on the line $y=1-2 x$. Hence, $v_{4}=3 / 2-v_{1} / 2$, which implies $v_{4}>4 / 3$. This, in turn, implies $x_{5}<x_{4}<3 / 4$. Based on these estimates, we observe that the portion of the line segment $y=3 / 2-2 x$ corresponding to $1 / 6 \leq y \leq$ $1 / 3$ is contained in $P^{\prime}$.

It follows from our assumption $C \cap P^{\prime}=\emptyset$ that $q<12$. Since $\left\{v_{4}\right\}=\frac{q-p}{2 q}$, we must have $p$ and $q$ odd. A look at Table 1 shows that the only cases in which $\left\{v_{4}\right\}=(q-p) / 2 q$ 


\begin{tabular}{|c|c|c|c|c|c|}
\hline \multicolumn{5}{|c|}{$(2,-1)_{S}$} \\
\hline$q$ & $p$ & & $q$ & $p$ & \\
\hline 5 & 1 & $\left(\frac{9}{10}, \frac{3}{10}\right)$ & 7 & 1 & -- \\
\hline 6 & 1 & -- & 7 & 2 & $\left(\frac{13}{14}, \frac{2}{7}\right)$ \\
\hline
\end{tabular}

\begin{tabular}{|c|c|c|c|c|c|}
\hline \multicolumn{6}{|c|}{$(2,-2)_{S}$} \\
\hline$q$ & $p$ & & $q$ & $p$ & \\
\hline 8 & 1 & -- & 10 & 3 & $\left(\frac{9}{10}, \frac{7}{20}\right)^{*}$ \\
\hline 10 & 1 & $\left(1, \frac{1}{4}\right)$ & 12 & 1 & $\left(\frac{25}{24}, \frac{5}{24}\right)^{* *}$ \\
\hline
\end{tabular}

\begin{tabular}{|c|c|c|c|c|c|c|c|c|}
\hline \multicolumn{10}{|c|}{$(5,1)_{U}$} \\
\hline$q$ & $p$ & & $q$ & $p$ & $q$ & $p$ & $p$ & \\
\hline 10 & 1 & $\left(\frac{3}{4}, \frac{7}{20}\right)^{* *}$ & 12 & 1 & $\left(\frac{25}{24}, \frac{5}{24}\right)^{* *}$ & 15 & 1 & $\left(\frac{5}{6}, \frac{1}{6}\right)$ \\
\hline 11 & 1 & $\left(\frac{13}{22}, \frac{7}{22}\right)^{* *}$ & 13 & 1 & -- & 16 & 1 & $\left(\frac{35}{32}, \frac{7}{32}\right)^{* *}$ \\
\hline 11 & 2 & -- & 14 & 1 & -- & 17 & 1 & $\left(\frac{35}{34}, \frac{7}{34}\right)^{* *}$ \\
\hline
\end{tabular}

\begin{tabular}{|c|c|c|c|c|c|c|c|c|}
\hline \multicolumn{10}{|c|}{$(5,-1)_{S}$} \\
\hline$q$ & $p$ & & $q$ & $p$ & $q$ & $p$ & $p$ & \\
\hline 10 & 1 & $\left(\frac{3}{4}, \frac{7}{20}\right)^{* *}$ & 16 & 3 & $\left(\frac{25}{32}, \frac{11}{32}\right)^{*}$ & 20 & 3 & $\left(\frac{7}{8}, \frac{13}{40}\right)$ \\
\hline 11 & 1 & -- & 17 & 2 & $\left(\frac{35}{34}, \frac{5}{17}\right)^{* *}$ & 21 & 4 & $\left(\frac{5}{6}, \frac{1}{3}\right)$ \\
\hline 11 & 2 & $\left(\frac{15}{22}, \frac{4}{11}\right)^{*}$ & 17 & 3 & $\left(\frac{15}{17}, \frac{11}{34}\right)$ & 22 & 3 & $\left(\frac{45}{44}, \frac{13}{44}\right)^{* *}$ \\
\hline 13 & 2 & $\left(\frac{25}{26}, \frac{4}{13}\right)$ & 19 & 2 & $\left(\frac{35}{38}, \frac{6}{19}\right)$ & 23 & 3 & $\left(\frac{20}{23}, \frac{15}{46}\right)$ \\
\hline 15 & 2 & $\left(\frac{5}{6}, \frac{1}{3}\right)$ & 19 & 3 & $\left(\frac{15}{19}, \frac{13}{38}\right)^{* *}$ & 23 & 4 & $\left(\frac{45}{46}, \frac{7}{23}\right)$ \\
\hline
\end{tabular}

\begin{tabular}{|c|c|c|c|c|c|c|c|c|}
\hline \multicolumn{10}{|c|}{$(2,-3)_{S}$} \\
\hline$q$ & $p$ & & $q$ & $p$ & $q$ & $p$ & $p$ & \\
\hline 11 & 1 & $\left(\frac{21}{22}, \frac{7}{22}\right)$ & 16 & 5 & $\left(\frac{11}{16}, \frac{7}{32}\right)$ & 19 & 2 & $\left(\frac{39}{38}, \frac{4}{19}\right)^{* *}$ \\
\hline 13 & 2 & $\left(\frac{25}{26}, \frac{4}{13}\right)$ & 17 & 1 & $\left(\frac{35}{34}, \frac{7}{34}\right)^{* *}$ & 19 & 5 & $\left(\frac{37}{38}, \frac{11}{38}\right)$ \\
\hline 14 & 1 & $\left(1, \frac{1}{4}\right)$ & 17 & 4 & $\left(\frac{33}{34}, \frac{5}{17}\right)$ & & & \\
\hline
\end{tabular}

\begin{tabular}{|c|c|c|c|c|c|c|c|c|}
\hline \multicolumn{10}{|c|}{$(4,3)_{U}$} \\
\hline$q$ & $p$ & & $q$ & $p$ & $q$ & $p$ & $p$ & \\
\hline 19 & 3 & $\left(\frac{15}{19}, \frac{13}{38}\right)^{* *}$ & 22 & 3 & $\left(1, \frac{1}{4}\right)$ & 25 & 3 & $\left(\frac{24}{25}, \frac{11}{50}\right)$ \\
\hline 19 & 6 & $\left(\frac{18}{19}, \frac{4}{19}\right)$ & 23 & 3 & $\left(\frac{24}{23}, \frac{13}{46}\right)^{* *}$ & 25 & 6 & $\left(\frac{19}{25}, \frac{8}{25}\right)$ \\
\hline 20 & 3 & $\left(\frac{9}{10}, \frac{7}{40}\right)$ & 23 & 6 & $\left(\frac{22}{23}, \frac{5}{23}\right)$ & & & \\
\hline
\end{tabular}

\begin{tabular}{|c|c|c|}
\hline \multicolumn{3}{|c|}{$(4,-3)_{U}$} \\
\hline$q$ & $p$ & \\
\hline 19 & 5 & $\left(\frac{13}{19}, \frac{9}{38}\right)$ \\
\hline
\end{tabular}


is possible are $q=5, p=1 ; q=9, p=1 ; q=11, p=1 ; q=11, p=3$. In these cases we find the points $\left(\frac{3}{5}, \frac{3}{10}\right),\left(\frac{2}{3}, \frac{1}{6}\right),\left(\frac{13}{22}, \frac{7}{22}\right)$, and $\left(\frac{7}{11}, \frac{5}{22}\right)$, respectively, in $C \cap P^{\prime}$.

Case 5.2.10. $(2,1)_{U}$

In this case the generator $\left(\left\{v_{4}\right\},\left\{v_{1}\right\}\right)$ is on the line $y=x / 2$, and hence $v_{4}=1+2 v_{1}$.

We first prove $C \cap P \neq \emptyset\left(P^{\prime}\right.$ will be handled below). Let $l$ be the intersection of the line $y=x / 2$ with $P$. We claim that the left end of $l$ is on the vertical side of $P$. This will follow from the fact that the lower-left corner of $P$ is below the line $y=x / 2$. The latter requires the inequality

$$
\frac{2}{3}-\frac{v_{4}}{3}\left(1-x_{5}\right)>2\left[\frac{1}{6}-\frac{v_{1}}{3}\left(1-x_{5}\right)\right]
$$

which is equivalent to

$$
\left(v_{4}-2 v_{1}\right)\left(1-x_{5}\right)<1,
$$

which holds because $v_{4}-2 v_{1}=1$ and $1-x_{5}<1$.

The right end of $l$ is either (a) on the horizontal upper side side of $P$ or (b) beyond the vertical line $x=4 / 3-v_{4} / 3$. We handle each case separately, showing in each that $C \cap P \neq \emptyset$ unless $q<6$.

In case (a) the (horizontal) length of $l$, which we denote $h(l)$, satisfies

$$
h(l)=\frac{v_{4}}{3}\left(1-x_{5}\right)+\frac{2 v_{2}}{3}\left(1-x_{5}\right)=\frac{v_{4}+2 v_{2}}{3}\left(1-x_{5}\right)=1-x_{5} .
$$

We have $v_{4}=1+2 v_{1} \geq(q+2) / q$, which implies $v_{5} \geq(q+3) / q$. Thus, $1-x_{5} \geq 3 /(q+3)$. So, we are guaranteed that $l$ contains a point of $C$ if $2 / q \leq 3 /(q+3)$, that is, if $q \geq 6$.

In case (b), we have

$$
h(l) \geq \frac{4}{3}-\frac{v_{4}}{3}-\left[\frac{2}{3}-\frac{v_{4}}{3}\left(1-x_{5}\right)\right]=\frac{2}{3}-\frac{v_{4} x_{5}}{3}>1 / 3,
$$

and again we are guaranteed that $l$ contains a point of $C$ if $2 / q \leq 1 / 3$, that is, if $q \geq 6$.

The only remaining case is $q=5, p=1$. In this case $\left(\frac{3}{5}, \frac{3}{10}\right) \in C \cap P$.

The conclusion that $C \cap P \neq \emptyset$ is compatible with our assumption only if $v_{1}<1 / 6$ and $C \cap P^{\prime}=\emptyset$. If so, the intersection $l^{\prime}$ of $l$ with $P^{\prime}$ must be properly contained in $l$, and therefore the right end of $l^{\prime}$ is on the horizontal line $y=5 / 12-v_{1} / 3$. It follows that the length of $l^{\prime}$ satisfies

$$
\begin{aligned}
h\left(l^{\prime}\right)=2\left[\frac{5}{12}-\frac{v_{1}}{3}\right]-\left[\frac{2}{3}-\frac{v_{4}}{3}\left(1-x_{5}\right)\right] & =\frac{1}{6}+\frac{v_{4}-2 v_{1}-v_{4} x_{5}}{3} \\
& =\frac{1}{6}+\frac{1-v_{4} x_{5}}{3} \\
& >\frac{1}{6} .
\end{aligned}
$$


Hence, $C \cap P^{\prime}=\emptyset$ implies $q<12$. Since $v_{1}<1 / 6$ this leaves us with the cases $q=7, \ldots, 11$ and $p=1$. In these cases (with one exception) the points $\left(\frac{5}{7}, \frac{5}{14}\right),\left(\frac{5}{8}, \frac{5}{16}\right),\left(\frac{5}{9}, \frac{5}{18}\right),\left(\frac{7}{10}, \frac{7}{20}\right)$, and $\left(\frac{7}{11}, \frac{7}{22}\right)$, respectively, are in $C \cap P^{\prime}$. The one exception is that $\left(\frac{5}{9}, \frac{5}{18}\right)$ is not in $P^{\prime}$ for $q=9$ and $v_{5}=12 / 9$. Note that when $q=9$ we have $v_{4}=11 / 9$ and $v_{5}=s / 9$ for some $s \geq 12$. The $x$-coordinate of the left side of $P^{\prime}$ is given by

$$
x=\frac{2}{3}-\frac{v_{4}}{3}\left(1-x_{5}\right)=\frac{2}{3}-\frac{11(s-9)}{27 s},
$$

which is less than $5 / 9$ for $s \geq 13$. If $s=12$ then the interval [39/22,11/6] is a 012345 -gap, contradicting our initial assumption.

Case 5.2.11. $(1,2)_{U}$

In this case the generator $\left(\left\{v_{4}\right\},\left\{v_{1}\right\}\right)$ is on the line $y=2 x$, and hence $v_{4}=1+v_{1} / 2$. This implies

$$
v_{4}<7 / 6
$$

In terms of $p$ and $q$ we have $v_{4}=\frac{2 q+p}{2 q}$, which implies that $p$ is even (and therefore $q$ is odd, $q \geq 7)$.

We first prove that $C \cap P \neq \emptyset$. Let $l$ be the intersection of the line $y=2 x-1$ with $P$. Note that the right end of $l$ is on the horizontal upper side of $P$. The left end of $l$ is either (a) on the vertical side of $P$ or $(b)$ on the horizontal lower side of $P$. We handle each case separately, showing that $C \cap P \neq \emptyset$ unless $q=7$.

In case (a) the (horizontal) length of $l$ satisfies

$$
h(l)=\frac{v_{4}}{3}\left(1-x_{5}\right)+\frac{v_{2}}{6}\left(1-x_{5}\right)=\frac{2+v_{1}+v_{2}}{6}\left(1-x_{5}\right)=\frac{1-x_{5}}{2} .
$$

Now, we may write $v_{5}=\frac{2 q+p+2 i}{2 q}$, where $i \geq 1$ is an integer. In order to guarantee that $l$ contains a point of $C$, it suffices if $\frac{1-x_{5}}{2} \geq \frac{1}{q}$, that is, if $p+2 i \geq 6$. The only remaining possibility is $p=2, i=1$. In this case the speeds are $2 / q,(q-2) / q, 1,(q+1) / q$, and $(q+2) / q$. Let $j$ be an integer such that $\frac{1}{6} \leq \frac{2 j+1}{q} \leq \frac{1}{3}$ (such an integer exists whenever $q \geq 9$ ). The time $t=j+1 / 2$ is in a 012345 -gap, contradicting our initial assumption.

If case (b) occurs, then we must have $\frac{v_{4}}{3}\left(1-x_{5}\right)>\frac{1}{12}$. Together with (54), this implies that $1-x_{5}>3 / 14$. The vertical length of $l$, which we denote $v(l)$, in case (b) satisfies

$$
v(l)=\frac{1}{6}+\frac{v_{1}}{3}\left(1-x_{5}\right)+\frac{v_{2}}{3}\left(1-x_{5}\right)=\frac{1}{6}+\frac{1-x_{5}}{3}>\frac{5}{21} .
$$

Since $2 / q \leq 5 / 21$ for $q \geq 9, l$ must contain a point of $C$.

The only remaining case is $q=7, p=2$. In this case $\left(\frac{9}{14}, \frac{2}{7}\right) \in C \cap P$.

The conclusion $C \cap P \neq \emptyset$ is compatible with our initial assumption only if $v_{1}<1 / 6$ and $C \cap P^{\prime}=\emptyset$. The right end of $l^{\prime}$, the intersection of $l$ with $P^{\prime}$, is on the horizontal line $y=\frac{5}{12}-\frac{v_{1}}{3}$. As to the left end, we consider again the two cases above. 
In case (a), the (horizontal) length of $l^{\prime}$ satisfies

$$
h\left(l^{\prime}\right)=\frac{v_{4}}{3}\left(1-x_{5}\right)+\frac{1}{2}\left(\frac{1}{12}-\frac{v_{1}}{3}\right)=\frac{1}{24}+\frac{1}{3}-\frac{v_{4} x_{5}}{3} \geq \frac{1}{24} .
$$

Thus $l^{\prime}$ must contain a point of $C$ if $q \geq 24$. The remaining cases are $q=13,15,17,19,21,23$ with $p=2$. In these cases the points $\left(\frac{17}{26}, \frac{4}{13}\right),\left(\frac{19}{30}, \frac{4}{15}\right),\left(\frac{23}{34}, \frac{6}{17}\right),\left(\frac{25}{38}, \frac{6}{19}\right),\left(\frac{27}{42}, \frac{6}{21}\right)$, and $\left(\frac{31}{46}, \frac{8}{23}\right)$, respectively, are in $C \cap P^{\prime}$.

In case (b), the (horizontal) length of $l^{\prime}$ is more than $1 / 12$, and since $q>12$ there must be a point of $C$ in $l^{\prime}$.

Case 5.2.12. $(1,1)_{U}$

This is Part 6 of the proof of Proposition 11, in which we have $v_{4}=1+v_{1}=(q+p) / q$. In this situation there does not necessarily exist a long 01234-gap; that is, $C$ does not usually intersect $P$. So, we resort to a completely different, two-part argument. We first consider times when runner 1 is at $1 / 6$, times of the form $t=x_{1}(k+1 / 6)$ where $k$ is a nonnegative integer.

Claim 19. Assume $v_{1}=p / q, v_{2}=(q-p) / q$ and $v_{4}=(q+p) / q$. If there exists a nonnegative integer $k$ satisfying

$$
\left[\left\{v_{4} x_{1}\left(k+\frac{1}{6}\right)+\delta\right\}=\frac{2}{3} \text { and } 0 \leq \delta<1\right] \Rightarrow \delta \leq \frac{1}{12}+\frac{q}{12 p}
$$

then there exists a 01234-gap of length at least $x_{4} / 6$.

Proof. Let $t=x_{1}(k+1 / 6)$, and let $\delta$ be as in (55). Furthermore, let $t^{\prime}$ be the first time at or after $t$ when runner 4 is at $2 / 3$; that is , $t^{\prime}=t+\delta x_{4}$.

We consider the interval $I:=\left[t^{\prime}, t^{\prime}+x_{4} / 6\right]$, which is clearly a subset of a 4 -gap. We first note that the position of runner 1 at time $t^{\prime}+x_{4} / 6$ is between $1 / 6$ and $u$, where $u$ obeys

$$
u=\frac{1}{6}+\left(\delta+\frac{1}{6}\right) x_{4} v_{1} \leq \frac{1}{6}+\left(\frac{1}{4}+\frac{q}{12 p}\right) \frac{p}{q+p}<\frac{5}{12} .
$$

It follows that $I$ is contained in a 0134-gap. It remains to consider runner 2. Since $v_{2}=v_{4}-2 v_{1}$, the position of runner 2 at time $t^{\prime}$ satisfies

$$
\begin{aligned}
\left\{v_{2} t^{\prime}\right\} & =\left\{\left\{v_{4} t^{\prime}\right\}-\left\{2 v_{1} t^{\prime}\right\}\right\} \\
& =\left\{2 / 3-\left\{2 v_{1} t\right\}-\left\{2 v_{1} \delta x_{4}\right\}\right\} \\
& \geq \frac{2}{3}-\frac{1}{3}-\frac{2 p}{q+p}\left(\frac{1}{12}+\frac{q}{12 p}\right)=\frac{1}{6} .
\end{aligned}
$$

Therefore, $I$ is contained in a 01234-gap.

We conclude from Claim 19 that if there exists a nonnegative integer $k$ for which (55) holds then $v_{5}<2 v_{4}$. To get a lower bound on $v_{5}$ we consider times when runner 1 is at $1 / 3$, times of the form $t=x_{1}(j+1 / 3)$. 
Claim 20. Assume $v_{1}=p / q, v_{2}=(q-p) / q, v_{4}=(q+p) / q$ and $\mathcal{B}^{\prime}=\mathbb{R}^{+}$. If there exists a nonnegative integer $j$ satisfying

$$
\left\{v_{4} x_{1}(j+1 / 3)\right\}=\left\{\frac{1}{3}+\frac{q}{3 p}+\frac{j q}{p}\right\} \in[5 / 6,1) \cup[0,1 / 6]
$$

then $v_{5}>2 v_{4}$.

Proof. Let $t=x_{1}(j+1 / 3)$, and consider the 4-block $I$ whose closure contains $t$. Since $\left\{t v_{2}\right\}=\left\{\left\{t v_{4}\right\}+1 / 3\right\}$ and $\left\{t v_{3}\right\}=\left\{\left\{t v_{4}\right\}-1 / 3\right\}, I$ is a subset of a 23-gap. Since $v_{1}<v_{4} / 4$ and $\left\{v_{1} t\right\}=1 / 3, I$ is in fact a subset of a 0123-gap. Therefore, runner 5 must cover both ends of $I$ and $v_{5}>2 v_{4}$.

So, if there exist nonnegative integers $k$ and $j$ satisfying (55) and (56), respectively, then we have a contradiction. Since the lengths of the intervals given in (55) and (56) are at least $1 / 3$ and in each case we are concerned with a set of $p$ equidistant points on the circle, we have a contradiction for $p \geq 3$. It remains to consider $p=1$ and $p=2$.

Suppose $p=2$. In this case $q$ is odd and $q \geq 7$. First note that the two points given in (56) are of the form $\frac{a}{6}, \frac{a+3}{6}$ for some integer $a$. One of these points lies in the specified interval, and $v_{5}>2 v_{4}$. On the other hand, the interval given in (55) has length at least $1 / 2$ for $q \geq 10$. Thus, we have $v_{5}<2 v_{4}$ unless $q=7$ or $q=9$. However, in each of these cases $v_{1}>1 / 6$ and the time $x_{1} / 2$ is in a 1234-gap of length greater than $x_{4} / 6$. Thus, for any $q$ we have $v_{5}<2 v_{4}$ and a contradiction.

The only remaining case is $p=1$. First note that for $q \geq 6$ there exists an integer $k$ satisfying (55), and, therefore, for $q \geq 6$ we have $v_{5}<2 v_{4}$. To achieve a lower bound on $v_{5}$ we use both Claim 20 and the following

Claim 21. Assume $v_{1}=1 / q, v_{2}=(q-1) / q, v_{4}=(q+1) / q$ and $\mathcal{B}^{\prime}=\mathbb{R}^{+}$. If there exists an integer $m$ satisfying

$$
\frac{m}{q} \in\left[\frac{1}{3}+\frac{1}{6 q}, \frac{5}{12}-\frac{1}{6 q}\right]
$$

then $v_{5} \geq 2 v_{4}$.

Proof. Consider the 3-block $I:=(m-1 / 6, m+1 / 6)$. At time $t=m$ runner 3 is at 0 while runners 1 and 4 are at $m / q$ and runner 2 is at $1-m / q$. It follows that $I$ is contained in a 0124-gap, and runner 5 must cover both endpoints of $I$. Thus, $v_{5}>2 v_{3}=2$.

Now, if $v_{5}=(2 q+1) / q$ then the position of runner 5 at time $t=m$ is also $m / q$. Thus, the time $m+1 / 6$ remains uncovered.

It follows from Claim 20 that $v_{5}>2 v_{4}$ if $q \equiv 2(\bmod 3)$. Furthermore, it follows from Claim 21 that $v_{5} \geq 2 v_{4}$ if $q \geq 16$ or $q \in\{10,13,15\}$. Thus, we have a contradiction unless $q \in\{5,6,7,9,12\}$. We consider these cases individually. In each case we set $v_{5}=s / q$.

Case 5.2.12.1. $q=5, p=1$ 
It follows from Claim 20 that $v_{5}>2 v_{4}$. Thus, $s \geq 13$. Note that since $v_{1} \geq 1 / 6$ the interval $\left(5 x_{1} / 12,7 x_{1} / 12\right)$ does not constitute a 01-block. Thus, $\left[5 / 2+x_{2} / 6,5 / 2+1 / 3\right]$ is a 01234-gap, and we can conclude that $v_{5}<8 / 3$. Therefore, $s=13$. However, for this set of speeds $\left[5 / 3+x_{4} / 6,5 / 3+1 / 6\right]$ is a 012345-gap.

Case 5.2.12.2. $q=6, p=1$

As in the previous case, $\left(5 x_{1} / 12,7 x_{1} / 12\right)$ does not constitute a 01-block. Therefore, $(3-1 / 6,3+1 / 6)$ is a 3 -block that is contained in the 0124-gap [3- $\left.x_{4} / 3,3+x_{4} / 3\right]$. It follows that $v_{5}>2 v_{3}$ and that $v_{5}<2 v_{4}$; that is, $s=13$. However, for this set of speeds the interval $\left[2+1 / 6,2+x_{2} / 6\right]$ is a 012345 -gap.

Case 5.2.12.3. $q=7, p=1$

In this case the interval $\left[7 / 6+x_{2} / 6,7 / 6+x_{4} / 2\right]$ is a 01234 -gap. It follows that $v_{5}<$ $48 / 35$. Therefore, we must have $v_{5}=9 / 7$. However, for this set of speeds, the interval $\left[7 / 6+x_{2} / 6,7 / 6+x_{5} / 3\right]$ is a 012345 -gap.

Case 5.2.12.4. $q=9, p=1$

Note that the time $t=3 / 2$ is in a 01234-gap. Since the position of runner 5 at this time is $s / 6$ we must have $6 \mid s$. However, when this is the case $\left[3 / 2+x_{5} / 6,3 / 2+x_{4} / 6\right]$ is a 012345-gap.

Case 5.2.12.5. $q=12, p=1$

In this case the interval $\left[2+x_{2} / 3,2+2 x_{4} / 3\right]$ is a 01234-gap. It follows that $s \in\{14,15\}$. If $s=14$ then the interval [ $\left.2+x_{2} / 3,2+x_{5} / 2\right]$ is a 012345-gap. On the other hand, if $s=15$ then $\left[2+2 x_{5} / 3,2+2 x_{4} / 3\right]$ is a 012345-gap.

\section{$6 \quad\left\{v_{1} t_{0}\right\} \neq 0,\left\{t_{0}\right\}=0$, and $x_{1}>3$}

Here we have $v_{3} t_{0}=t_{0}=k$ for some integer $k \geq 2$; in words, when runner 3 passes runner 2 both runners are at 0 . This implies $k=t_{0}=\frac{1}{1-v_{2}}$, and therefore we have

$$
v_{2}=\frac{k-1}{k} \quad \text { for some integer } \quad k \geq 2 .
$$

As Lemma 4 is not applicable in this case, we begin our discussion with a lemma that plays the role that Lemma 4 plays in Section 2.

Lemma 22. If there exists an interval $I$ and $t \in I$ such that $I \cap\left(B_{1} \cup B_{2} \cup B_{3}\right)=\emptyset$, $|I| \geq 1 / 3$ and $\left\{v_{4} t\right\}=1 / 2$ then $\mathcal{B} \neq \mathbb{R}^{+}$.

Proof. Clearly, $t$ lies in a 1234-gap. If $t+x_{4} / 3 \in I$ or $t-x_{4} / 3 \in I$ then the length of this 1234-gap is at least $x_{4} / 3$, and the result follows (since the length of any 5 -block is $x_{5} / 3$ ). If $t+x_{4} / 3, t-x_{4} / 3 \notin I$ then $I$ itself is contained in a 1234-gap. This 1234-gap has length at least $1 / 3>x_{5} / 3$. 
It remains to show that when (58) holds there exists an interval satisfying the conditions of Lemma 22. In order to do so, we consider the first 23-gap after times of the form $j t_{0}$. These are intervals of the form $\left[j t_{0}+x_{2} / 6, j t_{0}+5 / 6\right]$. It follows from (58) that $x_{2} \leq 2$ and therefore $I_{j}:=\left[j t_{0}+2 / 6, j t_{0}+5 / 6\right]$ does not intersect $B_{2} \cup B_{3}$. It is easy to see that if we have

$$
1 / 6-2 v_{1} / 6 \leq\left\{v_{1} j t_{0}\right\} \leq 5 / 6-5 v_{1} / 6
$$

then $I_{j} \cap B_{1}=\emptyset$. It follows from $x_{1}>3$ that (59) defines an interval in $[0,1)$ of length $4 / 6-3 v_{1} / 6>1 / 2$. Since $\left\{v_{1} t_{0}\right\} \neq 0$, there exists a $j$ satisfying (59) and hence there exists an $I_{j}$ that is the subset of a 123-gap (this observation is simply a one dimensional version of the observation given in (11)). Lemma 4 then implies that (3) holds if $x_{4} \leq 3 / 4$. So, we henceforth assume $x_{4}>3 / 4$. Under this assumption it is easy to see that if we have

$$
\left\{v_{4} j t_{0}\right\} \notin\left(\frac{1}{2}-\frac{v_{4}}{3}, \frac{3}{2}-\frac{5 v_{4}}{6}\right)
$$

then there exists $t \in I_{j}$ such that $\left\{v_{4} t\right\}=1 / 2$. Note that (60) excludes an interval on the circle $[0,1)$ of length $1-v_{4} / 2<1 / 2$.

We are now ready to apply the main lemma. Let $G$ be the additive subgroup of $T$ generated by $\left(\left\{v_{1} t_{0}\right\},\left\{v_{4} t_{0}\right\}\right)$ and define

$$
R=\left[1 / 6-v_{1} / 3,5 / 6-5 v_{1} / 6\right] \times\left[9 / 6-5 v_{4} / 6,9 / 6-2 v_{4} / 6\right] .
$$

Note that $R$ is a $\left(4 / 6-3 v_{1} / 6\right) \times 3 v_{4} / 6$ rectangle on the torus $T$. Note further that for any $x_{1}>3$ and any $x_{4}>3 / 4$ we have

$$
[1 / 6,5 / 9] \times[2 / 3,1] \subseteq R
$$

Now, it follows from Lemma 22, (59) and (60) that $G \cap R \neq \emptyset$ implies (3). Corollary 6 implies that $G \cap R \neq \emptyset$ unless one of the following conditions holds (note that we are using notation from Section 2).

1. $n_{1}=1$ or $n_{2}=1$

2. There exists $v \in\{ \pm 1 / n, \pm 2 / n\}$ such that $(1 / n, v) \in G$.

As usual, we handle these situations case by case. Note that we apply (14) in the statement of some of the cases.

Case 6.1. $n_{1}=1$ or $n_{2}=1$

First note that $n_{1}=1$ is impossible because we assume $\left\{v_{1} t_{0}\right\} \neq 0$. If $n_{2}=1$ then the circle $L_{0}^{2}$ has an intersection with $R$ of length $2 / 3-v_{1} / 2>1 / 2$ while the period of $G$ in $L_{0}^{2}$ is $1 / n_{1} \leq 1 / 2$. Thus it follows from (11) that $G \cap R \neq \emptyset$.

Case 6.2. $(1 / n, 1 / n) \in G$ and $n \geq 2$. 
In this case it is possible to have $G \cap R=\emptyset$. So, we are forced to use a different ad hoc argument here.

It follows from $n \geq 2$ that there exists $(u, u) \in G$ such that $1 / 3 \leq u \leq 1 / 2$. In other words, there exists an integer $j$ satisfying

$$
\gamma:=\left\{v_{1} j t_{0}\right\}=\left\{v_{4} j t_{0}\right\} \in[1 / 3,1 / 2] .
$$

Our goal here will be to show that for such a $j$ runner 5 cannot do all the things asked of it around the time $j t_{0}$. In order to do this we consider two subcases.

Case 6.2.1. $v_{2}=1 / 2$

We begin with a simple lemma.

Lemma 23. $[7 / 6,10 / 6] \nsubseteq B_{4} \cup B_{5}$.

Proof. Assume for the sake of contradiction that $[7 / 6,10 / 6] \subseteq B_{4} \cup B_{5}$. By Lemma 4 we have $x_{4}>3 / 4$. It follows that $11 x_{4}>33 / 4,[7 / 6,33 / 24] \subseteq B_{5}$ and $x_{5}>1 / 2$. Since $x_{5}>1 / 2$ the 45 -block containing $[7 / 6,10 / 6]$ consists of exactly one 4-block and exactly one 5-block. Thus, $11 x_{5}<7,11 x_{4}<13 x_{5}$ and $10<13 x_{4}$. These three inequalities yield

$$
\frac{10}{13}<x_{4}<\frac{13}{11} x_{5}<\frac{91}{121}
$$

This is an obvious contradiction.

Since $[7 / 6,10 / 6]$ is a 23 -gap in this case, it follows from Lemma 23 that $B_{1}$ must intersect $[7 / 6,10 / 6]$, and we must have $x_{1}>7$. Consider the time $j t_{0}$ defined above. Since $\left\{v_{1} j t_{0}\right\}=\gamma \in[1 / 3,1 / 2]$ the following define 123-gaps:

$$
\begin{gathered}
I_{1}=\left[j t_{0}-5 / 6, j t_{0}-2 / 6\right], \\
I_{2}=\left[j t_{0}+2 / 6, j t_{0}+5 / 6\right], \\
I_{3}=\left[j t_{0}+7 / 6, j t_{0}+10 / 6\right] .
\end{gathered}
$$

Since $\left\{v_{1} t_{0}\right\}=\left\{v_{4} t_{0}\right\}$, there exists a positive integer $m$ for which we have

$$
v_{4}-v_{1}=m / t_{0}=m\left(1-v_{2}\right)=m / 2 .
$$

It follows from $v_{4}<4 / 3$ and $v_{1}<1 / 7$ that $m=2, v_{4}=1+v_{1}$ and $v_{4}<8 / 7$.

Now, the 2-block separating $I_{1}$ from $I_{2}$ cannot contain a 4-block, because at its midpoint, $j t_{0}$, runner 4 is in $[1 / 3,1 / 2]$ and has speed $v_{4}<8 / 7$. It follows that the 4 -blocks meeting $I_{1}$ and $I_{2}$ are consecutive ones, which implies the inequality $8 x_{4}+4 x_{5}>10$, and in particular $x_{5}>1 / 2$.

Since $\left\{v_{4} j t_{0}\right\} \in[1 / 3,1 / 2]$ and $v_{4}<8 / 7$, runner 4 covers neither $j t_{0}+5 / 6$ nor $j t_{0}+7 / 6$. However, since $x_{5}>1 / 2$, runner 5 cannot cover both these points.

Case 6.2.2. $v_{2} \geq 2 / 3$ 
In this case we know the interval

$$
I=\left[j t_{0}+1 / 4, j t_{0}+5 / 6\right]
$$

is contained in a 123-gap. It follows immediately from Lemma 4 that $x_{4}>7 / 8$. Since the endpoints of $I$ cannot be covered by runner 4, the 45-block covering $I$ consists of a 4-block flanked by a 5 -block on either side, and $x_{5}<x_{4} / 2$.

On the other hand, if $n>2$ then there exists a $k$ such that $\left\{v_{1} k t_{0}\right\}=\left\{v_{4} k t_{0}\right\} \in$ $[1 / 6,1 / 3]$. Then the interval $\left[k t_{0}+1 / 4, k t_{0}+\left(5 / 6-\left\{v_{4} k t_{0}\right\}\right) x_{4}\right]$ is contained in a 1234-gap of length at least $x_{4} / 6$. This interval cannot be covered by runner 5 because $x_{5}<x_{4} / 2$. This leaves $n=2$, but in this case, $\left\{v_{1} t_{0}\right\}=1 / 2$ and the interval $\left[t_{0}+2 x_{4} / 3, t_{0}+5 / 6\right]$ is a 1234-gap of length at least 1/6. Again, this interval cannot be covered by runner 5 .

Case 6.3. $(1 / n,-1 / n) \in G$ and $n \geq 2$

It follows from (61) that if $1 / 6 \leq 1 / n \leq 1 / 3$ then $(1 / n,-1 / n) \in G \cap R$ and that if $1 / n<1 / 6$ then some multiple of $(1 / n,-1 / n)$ lies in $G \cap R$. This leaves us with the case $n=2$. However, when $n=2$ we have $1 / n=-1 / n$. So this case was handled above in Case 6.2.

Case 6.4. $(1 / n, 2 / n) \in G$ and $n \geq 4$

Here it follows from (61) that if there exists an integer $j$ such that $j / n \in[1 / 3,1 / 2]$ then $j(1 / n, 2 / n) \in G \cap R$. Such a $j$ exists for all $n$.

Case 6.5. $(1 / n,-2 / n) \in G$ and $n \geq 4$

Once again, we apply (61). In this case we conclude that if there exists $j$ such that $j / n \in[1 / 2,5 / 9]$ then $j(1 / n,-2 / n) \in G \cap R$. Such a $j$ exists unless $n \in\{5,7\}$.

Case 6.5.1. $n=5$

In this case $(1 / 5,3 / 5) \in G \cap R$ unless $3 / 5<9 / 6-5 v_{4} / 6$. So, we may assume

$$
x_{4}>25 / 27 \text {. }
$$

Similarly $(3 / 5,4 / 5) \in G \cap R$ unless $3 / 5>5 / 6-5 v_{1} / 6$, and we may assume

$$
x_{1}<25 / 7 \text {. }
$$

It follows that $\left[x_{1} / 6,5 x_{4} / 6\right]$ is a 1234-gap. Thus, there exists $j>0$ such that $(6 j-1) x_{5}<$ $x_{1}$ and $5 x_{4}<(6 j+1) x_{5}$. It then follows from (62) and (63) that $j=1$. We obtain

$$
\frac{125}{189}<\frac{5 x_{4}}{7}<x_{5}<\frac{x_{1}}{5}<\frac{5}{7}
$$

It follows that $t=7 / 6$ lies in a 2-block, and so we must have $x_{2}<7 / 5$. Next, the point $7 x_{2} / 6$ must be covered by a 5 -block, namely $\left(11 x_{5} / 6,13 x_{5} / 6\right)$. It is then the case that no block covers $t=13 x_{5} / 6$.

Case 6.5.2. $n=7$

Here we have $(1 / 7,5 / 7),(4 / 7,6 / 7) \in G$. It follows from $(61)$ and the fact that the width of $R$ is more than $1 / 2$ that either $(1 / 7,5 / 7) \in R$ or $(4 / 7,6 / 7) \in R$. 


\begin{tabular}{|c|c|c|c|c|c|c|c|c|c|}
\hline$(3,0)$ & $(4,0)$ & $(3,1)$ & $(3,-1)$ & $(4,1)$ & $(4,-1)$ & $(3,2)$ & $(3,-2)$ & $(4,2)$ & $(4,-2)$ \\
\hline $1 / 2$ & $2 / 3$ & $2 / 3$ & $2 / 3$ & $2 / 3$ & $1 / 2$ & $1 / 2$ & $7 / 18$ & $2 / 3$ & $8 / 15$ \\
\hline 6 & 8 & 6 & 6 & 8 & 8 & 11 & 11 & 14 & 14 \\
\hline
\end{tabular}

Table 9: Exceptional pairs in Section 7 for which (11) alone suffices to finish the proof.

\section{$7 \quad\left\{v_{1} t_{0}\right\} \neq 0,\left\{t_{0}\right\} \neq 0$, and $3 \leq x_{1}<4$}

Here we extend the argument given in Section 2, where we assumed $x_{1} \geq 4$. Again, we consider the subgroup $G$ of $T$ generated by $\left(\left\{t_{0}\right\},\left\{v_{1} t_{0}\right\}\right)$. By assumption, $G$ is not contained in one of the axes of $T$. As in Section 2, it suffices to show $G \cap P \neq \emptyset$, where $P$ is the parallelogram defined by (8) and (9). Since $P$ shrinks as $x_{1}$ gets smaller, we focus on the worst case in our domain, namely $x_{1}=3$. In this case $P$ is defined by

$$
\frac{1}{6} \leq x \leq \frac{5}{6}, \quad \frac{1}{9}+\frac{1}{3} x \leq y \leq \frac{5}{9}+\frac{1}{3} x .
$$

Thus, $P$ contains the rectangle $R=[1 / 6,5 / 6] \times[7 / 18,11 / 18]$ which has width $\alpha=2 / 3$ and height $\beta=2 / 9$.

By Corollary $6, G \cap R \neq \emptyset$ unless $G$ contains a minimal nonzero element of the form $(i / n, j / n)$ where $i$ and $j$ satisfy

$$
i \in\{0,1,2,3,4\} \quad \text { and } \quad|j|<\frac{3}{2}+\frac{1}{3} i .
$$

We take advantage of the full $P$ to show that, with two exceptions, $G \cap P \neq \emptyset$ even in these cases. First note that $(i, j) \in\{(0, \pm 1),(1,0)\}$ is ruled out by our assumption that $G$ is not contained in an axis of $T$. The cases where $(i, j) \in\{(2,0),(1, \pm 1),(2, \pm 2)\}$ were treated in Section 2, and the arguments used there apply here as well. This leaves us with 12 possible cases for the pair $(i, j)$.

In 10 of these cases there exists a circle $L$ (parallel to $L_{(i / n, j / n)}$ ) such that the length of $L \cap P$ exceeds or equals the period of $G$ in $L$. These cases are considered in Table 9 . In each column we consider a particular $(i, j)$. The second row contains the length of the intersection $L \cap P$, measured horizontally, and the third row contains the lower bound on $n$ given by (14). Since the period of $G$ in $L$ is $i / n$, we see that $G \cap P \neq \emptyset$ in each case.

We are left with the cases $(i, j)=(2,1)$ and $(i, j)=(2,-1)$. In each of these cases, it follows from (14) that we may assume $n \geq 4$. The second column in Table 10 gives the length of the longest intersection of $L_{(i / n, j / n)}$ with $P$. The values of $n$ for which this intersection length does not imply $G \cap P \neq \emptyset$ are considered in the other columns. In these columns we specify an element of $G \cap P$ if it exists. This leaves us with two exceptional groups which are considered below.

Consider the case $G=G_{4}$, the group generated by $(1 / 2,1 / 4)$. There exists a positive integer $k$ such that $\left\{k t_{0}\right\}=1 / 2$ and $\left\{k t_{0} v_{1}\right\}=1 / 4$. The interval

$$
I=\left[k t_{0}-x_{1} / 12, k t_{0}+1 / 3\right]
$$




\begin{tabular}{|c|c|c|c|c|c|c|c|c|c|}
\hline$(i, j)$ & & $n=4$ & $n=5$ & $n=6$ & $n=7$ & $n=8$ & $n=9$ & $n=10$ & $n=11$ \\
\hline$(2,1)$ & $\frac{1}{6}$ & $G_{4}$ & $\left(\frac{4}{5}, \frac{2}{5}\right)$ & $\left(\frac{2}{3}, \frac{1}{3}\right)$ & $G_{7}$ & $\left(\frac{3}{4}, \frac{3}{8}\right)$ & $\left(\frac{2}{3}, \frac{1}{3}\right)$ & $\left(\frac{4}{5}, \frac{2}{5}\right)$ & $\left(\frac{8}{11}, \frac{4}{11}\right)$ \\
\hline$(2,-1)$ & $\frac{3}{10}$ & $G_{4}$ & $\left(\frac{4}{5}, \frac{3}{5}\right)$ & $\left(\frac{2}{3}, \frac{2}{3}\right)$ & & & & & \\
\hline
\end{tabular}

Table 10: Treating the remaining exceptional pairs in Section 7.

is a 123-gap. Since $I \cap B_{4}$ has total length at most $1 / 3, I \cap B_{5}$ must have total length at least $x_{1} / 12$. Note that in order to cover the point $t=x_{1} / 6$ (the beginning of the first 123gap), we must have $x_{5}<x_{1} / 5$. Hence the length of a 5 -block is less than $x_{1} / 15$. Thus, the 45-block covering $I$ must consist of a 4-block flanked by two 5 -blocks. Furthermore, these two 5 -blocks must be consecutive (otherwise $x_{5}<x_{4} / 5<1 / 5$ and $2 x_{5} / 3<2 / 15<x_{1} / 12$ ). This gives the conditions

$$
4 x_{5} / 3>1 / 3+x_{1} / 12 \geq 7 / 12 \text { and } x_{4}>2 x_{5},
$$

which imply the bounds

$$
7 / 16<x_{5}<1 / 2 \text { and } 7 / 8<x_{4}<1 \text {. }
$$

Under these conditions, however, the interval $[2 / 3,35 / 48]$ remains uncovered.

The case $G=G_{7}$, the group generated by $(2 / 7,1 / 7)$, is treated similarly. Let $k$ be a positive integer such that $\left\{k t_{0}\right\}=4 / 7$ and $\left\{v_{1} k t_{0}\right\}=2 / 7$. Then the interval

$$
J=\left[k t_{0}-5 / 14, k t_{0}+11 / 42\right]
$$

is contained in a 123-gap (here we use $x_{1} \geq 3$ ). Out of the total length of $J$, which is $13 / 21$, runner 5 must cover more than $2 / 7$. As $x_{5}<x_{1} / 5<4 / 5$, one 5 -block does not suffice for this, and so $J$ must be covered by a 4 -block flanked by two 5 -blocks. As above, these two 5-blocks must be consecutive, yielding the bounds

$$
13 / 28<x_{5}<1 / 2 \text { and } 13 / 14<x_{4}<1 \text {. }
$$

Given these bounds, the interval $[2 / 3,65 / 84]$ remains uncovered.

\section{$8 \quad x_{1} \leq 3$}

Here we assume for the sake of contradiction that $x_{1} \leq 3$ and $\mathcal{B}=\cup_{i=1}^{5} B_{i}=\mathbb{R}^{+}$. We will make a series of observations concerning the way in which $\mathcal{B}$ covers small $t \in \mathbb{R}^{+}$. This series of observations will progressively restrict the possible values of $x_{1}, \ldots, x_{5}$ until we finally reach a contradiction. We begin by dividing the argument in cases.

Case 8.1. $x_{4}>5 / 7$ 
We begin with

$$
\left(x_{1}, x_{2}, x_{3}, x_{4}, x_{5}\right) \in[1,3] \times\left[1, x_{1}\right] \times\{1\} \times\left[\frac{5}{7}, 1\right] \times\left[0, x_{4}\right] .
$$

It follows immediately that $\left[x_{1} / 6,5 x_{4} / 6\right]$ is a 1234 -gap, so there exists an integer $j>0$ satisfying

$$
(6 j-1) x_{5}<x_{1} \text { and } 5 x_{4}<(6 j+1) x_{5},
$$

which implies

$$
\frac{25(6 j-1)}{7(6 j+1)}<x_{1}
$$

Since we are assuming $x_{1} \leq 3$ this implies that $j=1$. Thus, we have

$$
5 x_{5}<x_{1} \text { and } 5 x_{4}<7 x_{5}
$$

With this observation in hand, we now have

$$
\left(x_{1}, x_{2}, x_{3}, x_{4}, x_{5}\right) \in\left[\frac{125}{49}, 3\right] \times\left[1, x_{1}\right] \times\{1\} \times\left[\frac{5}{7}, \frac{21}{25}\right] \times\left[\frac{25}{49}, \frac{3}{5}\right] .
$$

We now consider the 13 -gap $[7 / 6,11 / 6]$. It is easy to see that $\left[\max \left\{7,13 x_{5}\right\} / 6,11 x_{4} / 6\right]$ is now a 1345-gap. This implies

$$
5 x_{2}<\max \left\{7,13 x_{5}\right\} \leq 39 / 5 \Rightarrow x_{2}<39 / 25
$$

and

$$
11 x_{4}<7 x_{2}
$$

It now follows from $(66)$ that $\left[\max \left\{13 x_{4}, 7 x_{2}\right\} / 6,11 / 6\right]$ is a 1234-gap, and therefore we have

$$
17 x_{5}<\max \left\{13 x_{4}, 7 x_{2}\right\} \text { and } 11<19 x_{5} .
$$

This leaves us with

$$
\left(x_{1}, x_{2}, x_{3}, x_{4}, x_{5}\right) \in\left[\frac{55}{19}, 3\right] \times\left[1, \frac{39}{25}\right] \times\{1\} \times\left[\frac{5}{7}, \frac{21}{25}\right] \times\left[\frac{11}{19}, \frac{3}{5}\right] .
$$

We now consider two subcases.

Case 8.1.1. $13 x_{4} \leq 7 x_{2}$

Here we have

$$
17 x_{5}<7 x_{2}
$$

which leaves us with

$$
\left(x_{1}, x_{2}, x_{3}, x_{4}, x_{5}\right) \in\left[\frac{55}{19}, 3\right] \times\left[\frac{187}{133}, \frac{39}{25}\right] \times\{1\} \times\left[\frac{5}{7}, \frac{21}{25}\right] \times\left[\frac{11}{19}, \frac{3}{5}\right] .
$$

the eleCtronic journal of combinatorics 8 (No. 2) (2001), \#R3 
We now move on to consideration of the interval $\left[13 / 6,5 x_{1} / 6\right]$, which is obviously a 123-gap. It follows from (65) that $25 x_{5}<5 x_{1}$ and from (68) that $13<23 x_{5}$. Therefore, we must have

$$
17 x_{4}<13 \text { and } 5 x_{1}<19 x_{4}
$$

and

$$
\left(x_{1}, x_{2}, x_{3}, x_{4}, x_{5}\right) \in\left[\frac{55}{19}, \frac{247}{85}\right] \times\left[\frac{187}{133}, \frac{39}{25}\right] \times\{1\} \times\left[\frac{5}{7}, \frac{13}{17}\right] \times\left[\frac{11}{19}, \frac{3}{5}\right] .
$$

Finally, we consider time close to $t=23 / 6$. It turns out that $\left[37 x_{5} / 6,23 / 6\right]$ is a 1235-gap, which implies

$$
29 x_{4}<37 x_{5} \text { and } 23<31 x_{4} .
$$

This contradicts (65) and (70) (i.e. we cannot simultaneously have $5 x_{5}<x_{1}, 5 x_{1}<19 x_{4}$ and $\left.29 x_{4}<37 x_{5}\right)$.

Case 8.1.2. $7 x_{2}<13 x_{4}$

Here we have

$$
17 x_{5}<13 x_{4} .
$$

Appealing to (67) and (72) we have

$$
\left(x_{1}, x_{2}, x_{3}, x_{4}, x_{5}\right) \in\left[\frac{55}{19}, 3\right] \times\left[\frac{2057}{1729}, \frac{39}{25}\right] \times\{1\} \times\left[\frac{187}{247}, \frac{21}{25}\right] \times\left[\frac{11}{19}, \frac{3}{5}\right] .
$$

We now consider the interval $\left[13 / 6,5 x_{1} / 6\right]$. As in Case 8.1.1, we have that $13<23 x_{5}$ and $25 x_{5}<5 x_{1}$. Moreover, $13<11 x_{2}$. Therefore, for $t=13 / 6$ to be covered we must have

$$
17 x_{4}<13 .
$$

This now implies (note that we use (72))

$$
\left(x_{1}, x_{2}, x_{3}, x_{4}, x_{5}\right) \in\left[\frac{55}{19}, 3\right] \times\left[\frac{2057}{1729}, \frac{39}{25}\right] \times\{1\} \times\left[\frac{187}{247}, \frac{13}{17}\right] \times\left[\frac{11}{19}, \frac{169}{289}\right] .
$$

Next, we consider the points $t=109 / 30$ and $t=26 / 6$. It can be checked that both points lie in 1345-gaps. Hence, runner 2 covers both. This, however, is impossible, as they are too far apart to be covered by the same 2-block (having length at most 13/25) and too close to be covered by distinct 2-blocks (the length of a 2-gap is at least 4114/5187).

Case 8.2. $x_{4} \leq 5 / 7$

As in case 8.1 we first consider the 123-gap $\left[x_{1} / 6,5 / 6\right]$. This gap is covered by $B_{4} \cup B_{5}$ in one of two ways. Either $5 x_{4}<x_{1}$ in which case we have $\left[7 x_{4} / 6,5 / 6\right] \subseteq B_{5}$ or $x_{1} \leq 5 x_{4}$ and $\left[x_{1} / 6,5 x_{4} / 6\right] \cup\left[7 x_{4} / 6,5 / 6\right] \subseteq B_{5}$. We consider these two possibilities separately.

Case 8.2.1. $5 x_{4}<x_{1}$ 
Here there exists $j>1$ satisfying

$$
(6 j-1) x_{5}<7 x_{4} \text { and } 5<(6 j+1) x_{5} \text {. }
$$

This implies

$$
x_{1}>\frac{25(6 j-1)}{7(6 j+1)} \geq \frac{275}{91}
$$

a contradiction.

Case 8.2.2. $x_{1} \leq 5 x_{4}$

In this case there exist $1<j<k$ satisfying

$$
(6 j-1) x_{5}<x_{1}, 5 x_{4}<(6 j+1) x_{5},(6 k-1) x_{5}<7 x_{4} \text { and } 5<(6 k+1) x_{5} \text {. }
$$

If $j+2 \leq k$ then $5 x_{4}<(6 j+1) x_{5}$ and $(6 k-1) x_{5}<7 x_{4}$ imply $10 x_{5}<2 x_{4}$. If this is the case, then all 45 -blocks have length less than $1 / 3$ while the 123 -gap $\left[x_{1} / 6,5 / 6\right]$ has length at least $1 / 3$. So, we have $k=j+1$, and

$$
(6 j-1) x_{5}<x_{1}, 5 x_{4}<(6 j+1) x_{5},(6 j+5) x_{5}<7 x_{4} \text { and } 5<(6 j+7) x_{5} \text {. }
$$

This implies

$$
\frac{5(6 j-1)}{6 j+7}<x_{1}
$$

which implies $j=2$. So, we actually have

$$
11 x_{5}<x_{1}, 5 x_{4}<13 x_{5}, 17 x_{5}<7 x_{4} \text { and } 5<19 x_{5}
$$

and

$$
\left(x_{1}, x_{2}, x_{3}, x_{4}, x_{5}\right) \in\left[\frac{55}{19}, 3\right] \times\left[1, x_{1}\right] \times\{1\} \times\left[\frac{85}{133}, \frac{39}{55}\right] \times\left[\frac{5}{19}, \frac{3}{11}\right] .
$$

It now follows that $7 / 6,21 / 12 \notin B_{4} \cup B_{5}$, and therefore we have

$$
5 x_{2}<7 \text { and } 7 x_{2}>21 / 2 \text {. }
$$

This is a contradiction.

\section{References}

[BW] U. Betke and J.M. Wills, Untere Schranken für zwei diophantische ApproximationsFunktionen, Monatsh. Math. 76, 214-217 (1972).

[BGGST] W. Bienia, L. Goddyn, P. Gvozdjak, A. Sebő, M. Tarsi. Flows, view obstructions and the lonely runner, JCTB 72, 1-9 (1998).

[C1] T.W. Cusick, View obstruction problems, Aequationes Math. 8, 165-170 (1972). 
[C2] T.W. Cusick, View obstruction problems in n-dimensional geometry, JCTA 16, 1-11 (1974).

[C3] T.W. Cusick, View obstruction problems II, Proc. Amer. Math. Soc. 84, 25-28 (1982).

[CP] T.W. Cusick and C. Pomerance, View obstruction problems III, Journal of Number Theory 19, 131-139 (1984).

[P] O. Perron, Irrationalzahlen, $2^{\text {nd }}$ edition, Chelsea Publishing Company, New York, 1951.

[S] P.D. Seymour, Nowhere-zero 6-flows, JCTB 30, 130-135 (1981).

[W] J.M. Wills, Zur simultanen homogenen diophantischen Approximation I, Monatsh. Math. 72, 254-263 (1968). 